\title{
A GENERALIZED INDEX THEOREM FOR MORSE-STURM SYSTEMS AND APPLICATIONS TO SEMI-RIEMANNIAN GEOMETRY*
}

\author{
F. GIANNONI ${ }^{\dagger}$, A. MASIELLO ${ }^{\ddagger}$, P. PICCIONE ${ }^{\S}$, AND D. V. TAUSK $₫$
}

\begin{abstract}
We prove an extension of the Index Theorem for Morse-Sturm systems of the form $-V^{\prime \prime}+R V=0$, where $R$ is symmetric with respect to a (non positive) symmetric bilinear form, and thus the corresponding differential operator is not self-adjoint. The result is then applied to the case of a Jacobi equation along a geodesic in a Lorentzian manifold, obtaining an extension of the Morse Index Theorem for Lorentzian geodesics with variable initial endpoints. Given a Lorentzian manifold $(\mathcal{M}, g)$, we consider a geodesic $\gamma$ in $\mathcal{M}$ starting orthogonally to a smooth submanifold $\mathcal{P}$ of $\mathcal{M}$. Under suitable hypotheses, satisfied, for instance, if $(\mathcal{M}, g)$ is stationary, the theorem gives an equality between the index of the second variation of the action functional $f$ at $\gamma$ and the sum of the Maslov index of $\gamma$ with the index of the metric $g$ on $\mathcal{P}$. Under generic circumstances, the Maslov index of $\gamma$ is given by an algebraic count of the $\mathcal{P}$-focal points along $\gamma$. Using the Maslov index, we obtain the global Morse relations for geodesics between two fixed points in a stationary Lorentzian manifold.
\end{abstract}

1. Introduction. The goal of this paper is to prove an index theorem for MorseSturm systems of differential equations with coefficients that are symmetric with respect to an indefinite inner product of $\mathbb{R}^{n}$. The main motivation for this kind of investigation comes from semi-Riemannian geometry, where Morse-Sturm systems appear in the form of Jacobi equations for vector fields along geodesics.

Let $(\mathcal{M}, g)$ be a semi-Riemannian manifold, $\mathcal{P}$ a smooth submanifold of $\mathcal{M}$ and $\gamma:[0,1] \mapsto \mathcal{M}$ be a geodesic in $\mathcal{M}$, with $\gamma(0) \in \mathcal{P}$ and $\dot{\gamma}(0) \in T_{\gamma(0)} \mathcal{P}^{\perp}$; set $q=\gamma(1)$. The curve $\gamma$ is then a stationary point of the action functional

$$
f(z)=\frac{1}{2} \int_{0}^{1} g(\dot{z}, \dot{z}) \mathrm{d} t,
$$

defined in the space $\Omega_{\mathcal{P}, q}$ of curves joining $\mathcal{P}$ and the point $q$ in $\mathcal{M}$. The index form $I_{\{\gamma, \mathcal{P}\}}$ is the symmetric bilinear form given by the second variation of $f$, defined on the tangent space $T_{\gamma} \Omega_{\mathcal{P}, q}$, which consists of vector fields $V$ along $\gamma$ with $V(0) \in T_{\gamma(0)} \mathcal{P}$ and $V(1)=0$. We recall the definition of $I_{\{\gamma, \mathcal{P}\}}$ :

$$
\begin{aligned}
I_{\{\gamma, \mathcal{P}\}}(V, W)= & \int_{0}^{1}\left[g\left(\nabla_{\dot{\gamma}} V, \nabla_{\dot{\gamma}} W\right)+g(R(\dot{\gamma}, V) \dot{\gamma}, W)\right] \mathrm{d} t \\
& -S_{\dot{\gamma}(0)}(V(0), W(0)),
\end{aligned}
$$

where $\nabla$ is the covariant derivative of the Levi-Civita connection of $g, R$ is the curvature tensor of $\nabla$ and $S_{\dot{\gamma}(0)}$ is the second fundamental form of $\mathcal{P}$ in the direction of $\dot{\gamma}(0)$.

\footnotetext{
${ }^{*}$ Received December 29, 1999; accepted for publication August 5, 2000.

†Dipartimento di Matematica e Fisica, Universitá di Camerino, Italy (giannoni@campus. unicam.it). Partially sponsored by M.U.R.S.T. (Italy).

$\ddagger$ Dipartimento Interuniversitario di Matematica, Politecnico di Bari, Italy (masiello@pascal. dm.uniba.it). Partially sponsored by M.U.R.S.T. (Italy).

$\S$ Departamento de Matemática, Universidade de São Paulo, Brazil (piccione@ime.usp.br). Partially sponsored by CNPq (Brazil), Processo 301410/95-0.

IDepartamento de Matemática, Universidade de São Paulo, Brazil (tausk@ime.usp.br). Sponsored by FAPESP (São Paulo, Brazil), Processo 98/12530-2.
} 
One obtains an infinite dimensional Hilbertian structure in $\Omega_{\mathcal{P}, q}$ by requiring a Sobolev $H^{1}$-regularity for the curves in $\Omega_{\mathcal{P}, q}$; then, $I_{\{\gamma, \mathcal{P}\}}$ is a bounded bilinear symmetric form on the Hilbert space $T_{\gamma} \Omega_{\mathcal{P}, q}$.

If $(\mathcal{M}, g)$ is Riemannian, i.e., if $g$ is a positive definite metric tensor, the celebrated Morse Index Theorem (see for instance [7, Theorem 2.2], [17, Theorem 15.1], [19] ) states that the index of $I_{\{\gamma, \mathcal{P}\}}$, which is the dimension of a maximal subspace of $T_{\gamma} \Omega_{\mathcal{P}, q}$ on which $I_{\{\gamma, \mathcal{P}\}}$ is negative definite, equals the geometric index $\mathrm{i}_{\text {geom }}(\gamma)$ of $\gamma$, which is the number of $\mathcal{P}$-focal points along $\gamma$ counted with multiplicity. Such equality can also be given in terms of the multiplicity of the negative eigenvalues of the Jacobi differential operator, which is a self-adjoint operator representing the index form in the Hilbert space of square-integrable vector fields along $\gamma$.

From the viewpoint of Calculus of Variations, the elements of $T_{\gamma} \Omega_{\mathcal{P}, q}$ are interpreted as infinitesimal variations of $\gamma$, and the index of $I_{\{\gamma, \mathcal{P}\}}$ on $T_{\gamma} \Omega_{\mathcal{P}, q}$ is the number of essentially different directions in which $\gamma$ can be deformed in order to obtain a curve of shorter length.

The theorem has been successively extended by Beem and Ehrlich to Lorentzian manifolds (see $[2,3]$ ), i.e., manifolds endowed with a metric tensor $g$ of index 1 , in the case of causal (non spacelike) geodesics. For such an extension one only needs minor modifications to the original statement (and proof) of the theorem. Most notably one needs to consider the restriction of $I_{\{\gamma, \mathcal{P}\}}$ to the space $T_{\gamma} \Omega_{\mathcal{P}, q}^{\perp}$ of vector fields along $\gamma$ which are pointwise orthogonal to $\gamma$. With this restriction, which in the Riemannian case is totally ininfluent for the computation of the index of $I_{\{\gamma, \mathcal{P}\}}$, one basically excludes the variations of $\gamma$ obtained by simple reparameterizations of $\gamma$. For timelike Lorentzian geodesics, the affine parameterization is the one that maximizes the value of the action functional, and thus the restriction to $T_{\gamma} \Omega_{\mathcal{\mathcal { P }}, q}^{\perp}$ has the effect of factoring out from $T_{\gamma} \Omega_{\mathcal{P}, q}$ an infinite dimensional space on which $I_{\{\gamma, \mathcal{P}\}}$ is negative definite, thus making the restricted $I_{\{\gamma, \mathcal{P}\}}$ into a form with finite index.

For spacelike Lorentzian geodesics, or more in general for geodesic of any causal character in semi-Riemannian manifolds with metrics of index greater than or equal to two, there is no hope to extend the original formulation of the index theorem, due mainly to the following reasons:

- the index of $I_{\{\gamma, \mathcal{P}\}}$ on both $T_{\gamma} \Omega_{\mathcal{P}, q}$ and $T_{\gamma} \Omega_{\mathcal{P}, q}^{\perp}$ is infinite;

- the set of $\mathcal{P}$-focal points along a geodesic may fail to be discrete, and there is no meaningful notion of geometric index;

- the Jacobi differential operator is no longer self-adjoint.

In the case of a geodesic $\gamma$ having only a finite number of $\mathcal{P}$-focal points, one can ask the question of whether there exists a natural subspace $\mathcal{K}^{\gamma}$ of $T_{\gamma} \Omega_{\mathcal{P}, q}$ with the property that the restriction of $I_{\{\gamma, \mathcal{P}\}}$ to $\mathcal{K}^{\gamma}$ has finite index, equal to the geometric index of $\gamma$. However, also for this special case the question seems to have a negative answer, due to the fact that, while the index of a bilinear form has some (semi-)continuity properties, the geometric index is not stable by small perturbations. Indeed, one can produce examples where (isolated) $\mathcal{P}$-focal points simply evaporate by arbitrary small perturbations of the metric (see [16]), or examples of a sequence $\gamma_{n}$ of geodesics having a finite number of $\mathcal{P}$-focal points converging to a geodesic $\gamma$ that has a continuum of $\mathcal{P}$-focal points (see [12]).

In order to prove an extension of the index theorem in semi-Riemannian geometry one needs to determine a natural subspace $\mathcal{K}^{\gamma}$ of the Hilbert space $T_{\gamma} \Omega_{\mathcal{P}, q}$ with the properties that:

- the index of the restriction of $I_{\{\gamma, \mathcal{P}\}}$ to $\mathcal{K}^{\gamma}$ is finite; 
- such index should be related to some geometrical properties of the geodesic $\gamma$ and of the manifold $\mathcal{P}$.

A hint for the choice of such a space was given by recent studies (see [10, 14]) concerning the geodesical connectedness of Lorentzian manifolds $(\mathcal{M}, g)$ whose metric $g$ is stationary, i.e., there exists a globally defined Killing timelike vector field on $\mathcal{M}$. Given any such vector field $Y$ on $\mathcal{M}$, one has a conservation law for geodesics given by:

$$
g(Y, \dot{\gamma}) \equiv c_{\gamma}(\text { constant })
$$

Considering the Hilbertian structure on $\Omega_{\mathcal{P}, q}$, one proves that the set $\Omega_{\mathcal{P}, q}^{Y}$ of curves in $\Omega_{\mathcal{P}, q}$ satisfying (1.2) almost everywhere is a smooth submanifold of $\Omega_{\mathcal{P}, q}$, and that the critical points of the restriction of the action functional $f$ to $\Omega_{\mathcal{P}, q}^{Y}$ are precisely the geodesics joining $\mathcal{P}$ and $q$ in $\mathcal{M}$. Given one such geodesic $\gamma$, the tangent space $\mathcal{K}^{\gamma}=T_{\gamma} \Omega_{\mathcal{P}, q}^{Y}$ is the Hilbert subspace of $T_{\gamma} \Omega_{\mathcal{P}, q}$ consisting of those vector fields $V$ along $\gamma$ that satisfy the linearization of (1.2). Using the Killing property of $Y$, the space $\mathcal{K}^{\gamma}$ can be described as:

$$
\mathcal{K}^{\gamma}=\left\{V \in T_{\gamma} \Omega_{\mathcal{P}, q}: g\left(\nabla_{\dot{\gamma}} V, Y\right)-g\left(V, \nabla_{\dot{\gamma}} Y\right) \equiv C_{V} \text { (constant) }\right\} .
$$

Using compact embeddings of the Sobolev space $H^{1}$ into the space $C^{0}$, one then proves that the restriction of the index form $I_{\{\gamma, \mathcal{P}\}}$ to $\mathcal{K}^{\gamma}$ is represented by a selfadjoint operator, which is a compact perturbation of the identity. In particular, its index is finite. The definition of the space $\mathcal{K}^{\gamma}$ makes perfectly sense also in the case that $Y$ is a timelike Jacobi field along $\gamma$, and also in this case we have finiteness of the index of the restriction of $I_{\{\gamma, \mathcal{P}\}}$ to $\mathcal{K}^{\gamma}$. Observe that the restriction of a Killing field along a geodesic is Jacobi, and thus this second construction is more general. This construction gives a solution for the first point mentioned in the program above; the next step is to give a geometrical interpretation of the value of the index of $I_{\{\gamma, \mathcal{P}\}}$ on $\mathcal{K}^{\gamma}$.

Inspired by some techniques in Hamiltonian systems (see [1]), it has recently been defined the notion of Maslov index for a semi-Riemannian geodesic (see [12] and also [16]), which is an integer number given by a certain topological invariant. Under generic circumstances, the Maslov index can be computed as a sort of algebraic count of the multiplicities of the $\mathcal{P}$-focal points. In particular, for Riemannian and causal Lorentzian geodesics it is always equal to the geometric index (see [16]). For spacelike Lorentzian geodesics, or more in general for all kinds of geodesics in semi-Riemannian manifolds with metric tensor of index greater or equal to two, the contribution of each $\mathcal{P}$-focal point to the value of the index is an integer number, possibly zero or negative, called the signature of the $\mathcal{P}$-focal point, whose absolute value is less than or equal to the multiplicity of the $\mathcal{P}$-focal point. Generically, the Maslov index of a semi-Riemannian geodesic is the sum of the signatures of its $\mathcal{P}$-focal points, and this sum is in absolute value less than or equal to the geometric index of the geodesic. Besides the finiteness, a remarkable property of the Maslov index is its stability by small perturbations (see [16]), due to its topological nature.

In this sense, the Maslov index of a geodesic is a natural candidate for substituting the notion of geometric index for Riemannian and causal Lorentzian geodesics.

The main result of the paper (Theorem 5.1 and its geometrical formulation Theorem 6.1) is that, if $\gamma(1)$ is not a $\mathcal{P}$-focal point along $\gamma$, then the index of the restriction of $I_{\{\gamma, \mathcal{P}\}}$ to $\mathcal{K}^{\gamma}$ is equal to the sum of the Maslov index of $\gamma$ and the index of the 
restriction of the Lorentzian metric $g$ to $T_{\gamma(0)} \mathcal{P}$. In particular, this number is independent on the choice of the vector field $Y$. To strengthen the analogy with the classical index Theorem, we remark that it was recently proven (see [16, Theorem 6.2.3]) that, under generic circumstances, the Maslov index of $\gamma$ is equal to the spectral index of $\gamma$, which is computed as a sort of algebraic count of the (real) negative eigenvalues of the Jacobi differential operator.

When comparing with the classical result of the Morse index theorem in Riemannian manifolds, we see that for non positive definite metrics some new phenomena appear:

- if $\mathcal{P}$ is timelike at $\gamma(0)$, i.e., if the restriction of $g$ to $T_{\gamma(0)} \mathcal{P}$ has positive index, then the initial value of the index of $I_{\{\gamma, \mathcal{P}\}}$ is strictly positive, hence even small portions of $\gamma$ are never local minimizers for the restricted action functional;

- each $\mathcal{P}$-focal point along $\gamma$ gives a contribution to the index which may be positive, negative or even null;

- the multiplicity of the $\mathcal{P}$-focal points is not stable by perturbations, and arbitrary small perturbations of a given geodesic may create or destroy focal points (see [16]).

By a parallel trivialization of the tangent bundle of $\mathcal{M}$ along the geodesic $\gamma$, one can reformulate the entire theory in terms of Morse-Sturm-Liouville systems of differential equations in $\mathbb{R}^{n}$. In this framework, the version of the Index Theorem discussed in this paper may be considered an extension of the Sturm Oscillation Theorem.

The proof of the main result of the paper is based on a general method for computing the variation of the index of a smooth curve $B(t)$ of symmetric bounded bilinear forms defined on a smooth family $\mathcal{H}_{t}$ of Hilbert spaces (Proposition 2.5). The jumps of the index function $i(t)=\operatorname{ind}\left(\left.B(t)\right|_{\mathcal{H}_{t}}\right)$ occur at the instants where $B(t)$ becomes singular, that correspond to the conjugate points. The value of the jump at a discontinuity point $t_{0}$ is then proven to be equal to the signature of the corresponding conjugate point (Proposition 3.5), under the assumption that the derivative $B^{\prime}\left(t_{0}\right)$ be non degenerate on $\operatorname{Ker}\left(B\left(t_{0}\right)\right)$. Under these circumstances, such calculation gives the proof of the aimed index Theorem.

Finally, we need to emphasize the fact that the stability of the Morse index and of the Maslov index (see [16]) plays a crucial role in the proof of our results. Namely, in order to employ the method described, we need to make a technical assumption concerning the non degeneracy of the restriction of $g$ to suitable subspaces. Such assumption, which holds generically, is needed to guarantee the finiteness of the set of conjugate points and it is the core of the proof of Proposition 2.5, where we show how to compute the jump of the index function at each conjugate point. The proof of the general case is then given using a perturbation argument, which is based on the observation that both the Morse index and the Maslov index of a semi-Riemannian geodesic do not change by small $C^{0}$-perturbations of the data.

Some examples and applications of the theory developed are discussed in the final part of the paper. In particular, under a suitable completeness assumption, we obtain the global Morse relations for geodesics with fixed endpoints in a stationary Lorentzian manifold (Theorem 7.2).

For a standard static Lorentzian manifold, the Morse relations have been proven in [4] using the Morse index of the energy functional restricted to the set of curves satisfying the constraint (1.2); the same kind of relations have been proven in [9] in the more general case of a standard stationary metric in a manifold with (possibly 
non smooth) convex boundary.

2. Abstract Results in Functional Analysis. Given Banach spaces $E_{1}$ and $E_{2}$, we denote by $\mathcal{L}\left(E_{1}, E_{2}\right)$ the set of all bounded linear operators from $E_{1}$ to $E_{2}$ and by $\mathrm{B}\left(E_{1}, E_{2}, \mathbb{R}\right)$ the set of all bounded bilinear maps from $E_{1} \times E_{2}$ to $\mathbb{R}$. If $E_{1}=$ $E_{2}=E$, we also set $\mathcal{L}(E)=\mathcal{L}(E, E)$ and $\mathrm{B}(E, \mathbb{R})=\mathrm{B}(E, E, \mathbb{R})$; by $\mathrm{B}_{\text {sym }}(E, \mathbb{R})$ we mean the set of symmetric bounded bilinear maps on $E$.

We give some general definitions concerning symmetric bilinear forms for later use.

DEFINITION 2.1. Let $V$ be any real vector space and $B: V \times V \mapsto \mathbb{R}$ a symmetric bilinear form. The negative type number (or index) $n_{-}(B)$ of $B$ is the possibly infinite number defined by

$$
n_{-}(B)=\sup \{\operatorname{dim}(W): W \text { subspace of } V \text { on which } B \text { is negative definite }\} .
$$

The positive type number $n_{+}(B)$ is given by $n_{+}(B)=n_{-}(-B)$; if at least one of these two numbers is finite, the signature $\operatorname{sgn}(B)$ is defined by:

$$
\operatorname{sgn}(B)=n_{+}(B)-n_{-}(B) .
$$

The kernel of $B, \operatorname{Ker}(B)$, is the set of vectors $v \in V$ such that $B(v, w)=0$ for all $w \in V$; the degeneracy $\operatorname{dgn}(B)$ of $B$ is the (possibly infinite) dimension of $\operatorname{Ker}(B)$.

If $V=V_{+} \oplus V_{-}$, where $B$ is positive semidefinite on $V_{+}$and negative definite on $V_{-}$, then $n_{-}(B)=\operatorname{dim}\left(V_{-}\right)$; for, obviously $n_{-}(B) \geq \operatorname{dim}\left(V_{-}\right)$and every subspace $S$ on which $B$ is negative definite satisfies $S \cap V_{+}=\{0\}$, and therefore $\operatorname{dim}(S) \leq \operatorname{dim}\left(V_{-}\right)$. Moreover, if in addition $B$ is positive definite on $V_{+}$, then $\operatorname{Ker}(B)=\{0\}$. Namely, if $v=v_{+}+v_{-} \in \operatorname{Ker}(B)$, with $v_{+} \in V_{+}$and $v_{-} \in V_{-}$, then, by considering the equality $-B\left(v_{+}, v_{-}\right)=B\left(v_{+}, v_{+}\right)=B\left(v_{-}, v_{-}\right)$, we get $v_{+}=v_{-}=0$. A simple density argument shows that if the symmetric bilinear form $B$ is continuous with respect to some norm in the vector space $V$, then its index does not change when one extends $B$ to the Banach space completion of $V$.

If $V$ is finite dimensional, then the numbers $n_{+}(B), n_{-}(B)$ and $d g n(B)$ are respectively the number of 1's, -1 's and 0's in the canonical form of $B$ as given by the Sylvester's Inertia Theorem. In this case, $n_{+}(B)+n_{-}(B)$ is equal to the codimension of $\operatorname{Ker}(B)$, and it is also called the $\operatorname{rank}$ of $B, \operatorname{rk}(B)$.

Given a Hilbert space $\mathcal{H}$ with inner product $\langle\cdot, \cdot\rangle$, to any bounded bilinear form $B: \mathcal{H} \times \mathcal{H} \mapsto \mathbb{R}$ by Riesz's theorem there corresponds a bounded linear operator $T_{B}: \mathcal{H} \mapsto \mathcal{H}$, which is related to $B$ by:

$$
B(x, y)=\left\langle T_{B}(x), y\right\rangle, \quad \forall x, y \in \mathcal{H}
$$

We say that $T_{B}$ is the linear operator associated to $B$ with respect to the inner product $\langle\cdot, \cdot\rangle$. Clearly, $B$ is symmetric if and only if $T_{B}$ is self-adjoint. We say that $B$ is non degenerate if $T_{B}$ is injective; $B$ will be said to be strongly non degenerate if $T_{B}$ is an isomorphism. If $T_{B}$ is a Fredholm operator of index 0, i.e., if $T_{B}$ is a compact perturbation of an isomorphism, then, by the Fredholm's Alternative, $B$ is non degenerate if and only if it is strongly non degenerate. Observe that the strong non degeneracy is stable by small perturbations, since the set of isomorphisms of $\mathcal{H}$ is open in $\mathcal{L}(\mathcal{H})$. 
We now give a criterion for the differentiability of curves in Banach spaces. We start with a definition

Definition 2.2. Let $E$ and $E_{0}$ be real Banach spaces. $A$ subset $\Phi \subset \mathcal{L}\left(E, E_{0}\right)$ is said to be separating for $E$ if for all $x \in E \backslash\{0\}$ there exists $\phi \in \Phi$ such that $\phi(x) \neq 0$.

We now prove the following:

Lemma 2.3. Let $E, E_{0}$ be real Banach spaces and $F, G:[a, b] \mapsto E$ be fixed maps, with $G$ continuous. Let $\Phi \subset \mathcal{L}\left(E, E_{0}\right)$ be a separating set for $E$; assume that for each $\phi \in \Phi$ the composition $\phi \circ F:[a, b] \mapsto E_{0}$ is of class $C^{1}$, and that $(\phi \circ F)^{\prime}(t)=\phi \circ G(t)$ for all $t \in[a, b]$. Then, $F$ is a map of class $C^{1}$, and $F^{\prime}(t)=G(t)$ for all $t \in[a, b]$.

Proof. Fix $t \in[a, b]$; we have to prove that $F^{\prime}(t)=G(t)$. We claim that the following equality holds:

$$
F(t+h)-F(t)=\int_{t}^{t+h} G(s) \mathrm{d} s
$$

It follows easily by applying each element $\phi \in \Phi$ to both sides of (2.3) and using the separating property of $\Phi$. Denoting by $\|\cdot\|$ the norm of $E$, it follows:

$$
\left\|\frac{F(t+h)-F(t)}{h}-G(t)\right\| \leq\left|\frac{1}{h} \int_{t}^{t+h}\|G(s)-G(t)\| \mathrm{d} s\right| ;
$$

the continuity of $G$ concludes the argument.

In the next proposition and its corollary we exhibit a method to compute the variation of the index of a curve of symmetric bilinear forms. We want to leave the domains of the forms variable, and we use the following notion of a $C^{1}$-curve of closed subspaces of a Hilbert space:

DEFINITION 2.4. Let $\mathcal{H}$ be a Hilbert space, $I \subset \mathbb{R}$ an interval and $\left\{\mathcal{D}_{t}\right\}_{t \in I}$ be a family of closed subspaces of $\mathcal{H}$. We say that $\left\{\mathcal{D}_{t}\right\}_{t \in I}$ is a $C^{1}$-family of subspaces if for all $t_{0} \in I$ there exists a $C^{1}$-curve $\left.\alpha:\right] t_{0}-\varepsilon, t_{0}+\varepsilon[\cap I \mapsto \mathcal{L}(\mathcal{H})$ and a closed subspace $\overline{\mathcal{D}} \subset \mathcal{H}$ such that $\alpha(t)$ is an isomorphism and $\alpha(t)\left(\mathcal{D}_{t}\right)=\overline{\mathcal{D}}$ for all $t$.

We will call the maps $\alpha$ appearing in Definition 2.4 the local trivializations of the family $\left\{\mathcal{D}_{t}\right\}_{t \in I}$.

In the following Proposition we study how the index of a smooth curve $B(t)$ of symmetric bilinear forms varies after passing through a degenerate instant $t_{0}$. We need a technical assumption on the map $B\left(t_{0}\right)$, which must be represented by a compact perturbation of a positive operator.

Proposition 2.5. Let $\mathcal{H}$ be a real Hilbert space with inner product $\langle\cdot, \cdot\rangle$, and let $B:\left[t_{0}, t_{0}+r\right] \mapsto \mathrm{B}_{\mathrm{sym}}(\mathcal{H}, \mathbb{R}), r>0$, be a map of class $C^{1}$. Let $\left\{\mathcal{D}_{t}\right\}_{t \in\left[t_{0}, t_{0}+r\right]}$ be a $C^{1}$-family of closed subspaces of $\mathcal{H}$, and denote by $\bar{B}(t)$ the restriction of $B(t)$ to $\mathcal{D}_{t} \times \mathcal{D}_{t}$. Assume that the following three hypotheses are satisfied:

1. $\bar{B}\left(t_{0}\right)$ is represented by an operator of the form $L+K$, with $L: \mathcal{D}_{t_{0}} \mapsto \mathcal{D}_{t_{0}}$ a positive isomorphism and $K: \mathcal{D}_{t_{0}} \mapsto \mathcal{D}_{t_{0}}$ a (self-adjoint) compact operator;

2. the restriction $\widetilde{B}$ of the derivative $B^{\prime}\left(t_{0}\right)$ to $\operatorname{Ker}\left(\bar{B}\left(t_{0}\right)\right) \times \operatorname{Ker}\left(\bar{B}\left(t_{0}\right)\right)$ is non degenerate;

3. $\operatorname{Ker}\left(\bar{B}\left(t_{0}\right)\right) \subseteq \operatorname{Ker}\left(B\left(t_{0}\right)\right)$. 
Then, for $t>t_{0}$ sufficiently close to $t_{0}, \bar{B}(t)$ is non degenerate, and we have:

$$
n_{-}(\bar{B}(t))=n_{-}\left(\bar{B}\left(t_{0}\right)\right)+n_{-}(\widetilde{B}),
$$

all the terms of the above equality being finite natural numbers.

Proof. By possibly passing to a smaller $r$, we can assume the existence of a $C^{1}$ curve $\alpha(t)$ of isomorphisms of $\mathcal{H}$ such that $\alpha(t)$ carries $\mathcal{D}_{t}$ to a fixed subspace $\overline{\mathcal{D}}$ of $\mathcal{H}$. We can now replace each $B(t)$ by the push-forward $B(t)\left(\alpha(t)^{-1} \cdot \alpha(t)^{-1} \cdot\right)$, and each $\mathcal{D}_{t}$ by $\overline{\mathcal{D}}$. Such replacements will not affect the hypotheses of the Proposition, nor the quantities involved in the equality (2.4). For instance, thanks to the hypothesis 3 , the index of the restriction of $B^{\prime}\left(t_{0}\right)$ to $\operatorname{Ker}\left(\bar{B}\left(t_{0}\right)\right)$ does not change; namely, for $V, W \in \operatorname{Ker}\left(\left.B\left(t_{0}\right)\left(\alpha\left(t_{0}\right)^{-1} \cdot, \alpha\left(t_{0}\right)^{-1} \cdot\right)\right|_{\overline{\mathcal{D}} \times \overline{\mathcal{D}}}\right)$, it is:

$$
\begin{aligned}
& \left.\frac{\mathrm{d}}{\mathrm{d} t} B(t)\left(\alpha(t)^{-1} V, \alpha(t)^{-1} W\right)\right|_{t=t_{0}}=B^{\prime}\left(t_{0}\right)\left(\alpha\left(t_{0}\right)^{-1} V, \alpha\left(t_{0}\right)^{-1} W\right) \\
& +\left.B\left(t_{0}\right)\left(\frac{\mathrm{d}}{\mathrm{d} t} \alpha(t)^{-1} V, \alpha\left(t_{0}\right)^{-1} W\right)\right|_{t=t_{0}}+\left.B\left(t_{0}\right)\left(\alpha\left(t_{0}\right)^{-1} V, \frac{\mathrm{d}}{\mathrm{d} t} \alpha(t)^{-1} W\right)\right|_{t=t_{0}} \\
& =B^{\prime}\left(t_{0}\right)\left(\alpha\left(t_{0}\right)^{-1} V, \alpha\left(t_{0}\right)^{-1} W\right) .
\end{aligned}
$$

We can therefore assume without loss of generality that $\mathcal{D}_{t}=\mathcal{H}$ and $\bar{B}(t)=B(t)$ for all $t$. Moreover, we observe here that, by a convenient choice of the Hilbert space inner product on $\mathcal{H}$, we can assume that $\bar{B}\left(t_{0}\right)=B\left(t_{0}\right)$ is represented by a compact perturbation of the identity of $\mathcal{H}, \mathrm{Id}+K$.

Now, the subspace $N=\operatorname{Ker}\left(B\left(t_{0}\right)\right)$ is the eigenspace of $K$ corresponding to the eigenvalue -1 , hence it is finite dimensional.

We start considering the case that $B\left(t_{0}\right)$ is positive semi-definite on $\mathcal{H}$ and that $\widetilde{B}$ is positive definite on $N$. In this case, the thesis means that $B(t)$ is positive definite on $\mathcal{H}$ for $t>t_{0}$ sufficiently close to $t_{0}$.

Let $S$ be any closed complementary subspace of $N$ in $\mathcal{H}$; clearly $B\left(t_{0}\right)$ is positive definite on $S$. We claim that there exists a positive constant $c_{0}$ such that, for $t$ sufficiently close to $t_{0}$, it is:

$$
B(t)[x, x] \geq c_{0}, \quad \forall x \in S \text { with }\|x\|=1 .
$$

Namely, for $t=t_{0}$, the inequality (2.6) follows from the fact that the restriction of $B\left(t_{0}\right)$ to $S$ is of the form $\langle(\operatorname{Id}+\bar{K}) \cdot, \cdot\rangle$ for some compact operator $\bar{K}: S \mapsto S$. In this case, $c_{0}$ may be chosen to be the least eigenvalue of $\mathrm{Id}+\bar{K}$. The continuity of $B$ concludes the proof of the claim.

We set:

$$
c_{1}=\inf _{\substack{y \in N \\\|y\|=1}} B^{\prime}\left(t_{0}\right)[y, y]>0
$$

Since $B$ is $C^{1}$, it is easy to see that, for $t$ sufficiently close to $t_{0}$, it is:

$$
B(t)[y, y] \geq \frac{1}{2} c_{1}\left(t-t_{0}\right), \quad \forall y \in N,\|y\|=1,
$$

so that $B(t)$ is positive definite on both $N$ and $S$ for $t$ sufficiently close to $t_{0}$. We want to show that, if $t>t_{0}$ is sufficiently close to $t_{0}$, then for all $x \in S \backslash\{0\}$ and $y \in N \backslash\{0\}, B(t)$ is positive definite on the two dimensional subspace of $\mathcal{H}$ generated 
by $x$ and $y$. By the positivity on $S$ and $N$, it suffices to prove that, for $t>t_{0}$ is sufficiently close to $t_{0}$, the following inequality holds:

$$
B(t)[x, y]^{2}<B(t)[x, x] \cdot B(t)[y, y]
$$

for all $x \in S, y \in N, x, y \neq 0$. Obviously, we can assume $\|x\|=\|y\|=1$. As $B\left(t_{0}\right)$ vanishes on $N \times S$ and $B$ is of class $C^{1}$, there exists $c_{2}>0$ such that, for all $t>t_{0}$ is sufficiently close to $t_{0}$, we have:

$$
|B(t)[x, y]| \leq c_{2} \cdot\left(t-t_{0}\right)
$$

for all $x \in S, y \in N$ with $\|x\|=\|y\|=1$. By (2.7), (2.8) and (2.10), for all $t>t_{0}$ is sufficiently close to $t_{0}$ we get:

$$
B(t)[x, y]^{2} \leq c_{2}^{2}\left(t-t_{0}\right)^{2}<\frac{1}{2} c_{0} c_{1}\left(t-t_{0}\right) \leq B(t)[x, x] \cdot B(t)[y, y],
$$

for all $x \in S, y \in N$ with $\|x\|=\|y\|=1$. This yields (2.9) and concludes the first part of the proof.

For the general case, we use the spectral decomposition of $K$ to write an orthogonal decomposition $\mathcal{H}=S_{+} \oplus S_{-} \oplus N$, where $B\left(t_{0}\right)$ is positive definite on $S_{+}$and negative definite on $S_{-}$; observe that $S_{-}$is finite dimensional, and $n_{-}\left(B\left(t_{0}\right)\right)=\operatorname{dim}\left(S_{-}\right)$. Moreover, we write $N=N_{+} \oplus N_{-}$, where $B^{\prime}\left(t_{0}\right)$ is positive definite on $N_{+}$and negative definite on $N_{-}$. We then apply the result proven in the first part of the proof to the restriction of $B(t)$ to $S_{+} \oplus N_{+}$once, and again to the restriction of $-B(t)$ to $S_{-} \oplus N_{-}{ }^{1}$. The conclusion follows by observing that $B(t)$ is positive definite on $S_{+} \oplus N_{+}$and negative definite on $S_{-} \oplus N_{-}$, which implies that $n_{-}(B(t))=\operatorname{dim}\left(S_{-} \oplus N_{-}\right)$for $t$ sufficiently close to $t_{0}$. Clearly, this also implies that $B(t)$ is non degenerate.

Although we will not need it, we observe that, for $t$ sufficiently close to $t_{0}$, the bilinear map $\bar{B}(t)$ is actually strongly non degenerate, as it follows easily from Fredholm's Alternative. We also observe that the assumption that the bilinear map $\bar{B}\left(t_{0}\right)$ be represented by a compact perturbation of a positive operator cannot be removed from the statement of Proposition 2.5; it is easy to give examples where the hypothesis is not satisfied and the thesis of Proposition 2.5 does not hold.

REMARK 2.6. It is important to emphasize that the conclusion of Proposition 2.5 does not hold if the assumption of nondegeneracy for the derivative $B^{\prime}\left(t_{0}\right)$ is not satisfied, and this is trivially checked. Besides, unless the Hilbert space $\mathcal{H}$ is onedimensional, it is very unlikely that the conclusion of Proposition 2.5 can be extended if one only makes a non degeneracy assumption for some higher order derivative $B^{(k)}\left(t_{0}\right)$ on $\operatorname{Ker}\left(B\left(t_{0}\right)\right)$; to understand this, we consider the following example. Let $B_{1}(t)$ and $B_{2}(t)$ be the symmetric bilinear forms on $\mathbb{R}^{2}$ represented with respect to the canonical basis by the following matrices:

$$
B_{1}(t)=\left(\begin{array}{cc}
t^{2} & t \\
t & 1+t
\end{array}\right), \quad B_{2}(t)=\left(\begin{array}{cc}
t^{2} & 0 \\
0 & 1
\end{array}\right) .
$$

Clearly, $t_{0}=0$ is an isolated singularity for both $B_{1}$ ad $B_{2}$, and $\operatorname{Ker}\left(B_{1}(0)\right)=$ $\operatorname{Ker}\left(B_{2}(0)\right)=\mathbb{R} \cdot e_{1}$, where $e_{1}$ is the first vector of the canonical basis of $\mathbb{R}^{2}$. The

\footnotetext{
${ }^{1}$ observe that $S_{-} \oplus N_{-}$has finite dimension, hence it is trivial that the restriction of $-B(t)$ to $S_{-} \oplus N_{-}$is represented by a compact perturbation of a positive isomorphism, say the identity, and the first part of the proof applies.
} 
derivatives $B_{1}^{\prime}(0)$ and $B_{2}^{\prime}(0)$ vanish on $\mathbb{R} \cdot e_{1}$; moreover, the restrictions of $B_{1}(t)$ and $B_{2}(t)$ on $\mathbb{R} \cdot e_{1}$ coincide for all $t$. However, the change of value of the functions $n_{-}\left(B_{1}(t)\right)$ and $n_{-}\left(B_{2}(t)\right)$ passing from a negative to a positive value of $t$ is different:

$$
\begin{array}{lll}
n_{-}\left(B_{1}(t)\right)=1, & \mathrm{n}_{-}\left(B_{2}(t)\right)=0, & \text { for } t<0, \\
n_{-}\left(B_{1}(t)\right)=0, & \mathrm{n}_{-}\left(B_{2}(t)\right)=0, & \text { for } t>0 .
\end{array}
$$

Remark 2.7. Observe that, under the hypotheses of Proposition 2.5, if $B(t)$ is non degenerate for $t$ in some interval $I$, then the function $i(t)=n_{-}(B(t))$ is constant on $I$. We also observe that Proposition 2.5 can be applied to a backwards reparameterization of the curve $B(t)$ to obtain information about the value of $n_{-}(B(t))$ for $t<t_{0}$ sufficiently close to $t_{0}$. Namely, if one considers the curve of bilinear maps $S(t)=B\left(t_{0}-t\right)$, we have $S(0)=B\left(t_{0}\right), S^{\prime}(0)=-B^{\prime}\left(t_{0}\right)$, and the equality (2.4) tells us that, for $\tau>0$ sufficiently small, it is:

$$
\begin{aligned}
n_{-}\left(\bar{B}\left(t_{0}-\tau\right)\right) & =n_{-}(S(\tau))=n_{-}\left(\bar{B}\left(t_{0}\right)\right)+n_{-}(-\widetilde{B}) \\
& =n_{-}\left(\bar{B}\left(t_{0}\right)\right)+n_{+}(\widetilde{B}) .
\end{aligned}
$$

We also have the following immediate corollary, which gives us a way to compute the total change of index of a differentiable curve of symmetric bilinear forms when passing through a degenerate instant:

Corollary 2.8. Let $B:\left[t_{0}-r, t_{0}+r\right] \mapsto \mathrm{B}_{\mathrm{sym}}(\mathcal{H}, \mathbb{R})$ and $\left\{\mathcal{D}_{t}\right\}_{t \in\left[t_{0}-r, t_{0}+r\right]}$ satisfy the same hypotheses of Proposition 2.5. Then, in the notations of Proposition 2.5, for $\varepsilon>0$ small enough, we have:

$$
n_{-}\left(\bar{B}\left(t_{0}-\varepsilon\right)\right)-n_{-}\left(\bar{B}\left(t_{0}+\varepsilon\right)\right)=\operatorname{sgn}(\widetilde{B}) .
$$

Proof. Use Proposition 2.5 twice, once to $\left.B\right|_{\left[t_{0}, t_{0}+r\right]}$ and once to a backwards reparameterization of $\left.B\right|_{\left[t_{0}-r, t_{0}\right]}$ (see Remark 2.7). $\square$

We conclude the section by showing a method that will be used later to produce $C^{1}$-families of closed subspaces of a Hilbert space:

Lemma 2.9. Let $I \subset \mathbb{R}$ be an interval, $\mathcal{H}, \tilde{\mathcal{H}}$ be Hilbert spaces and $F: I \mapsto$ $\mathcal{L}(\mathcal{H}, \tilde{\mathcal{H}})$ be a $C^{1}$-map such that each $F(t)$ is surjective. Then, the family $\mathcal{D}_{t}=$ $\operatorname{Ker}(F(t))$ is a $C^{1}$-family of closed subspaces of $\mathcal{H}$.

Proof. We exhibit local trivializations for the family $\left\{\mathcal{D}_{t}\right\}_{t \in I}$. For $t=t_{0} \in I$, the map $F(t)$ maps the orthogonal complement $\mathcal{D}_{t_{0}}^{\perp}$ isomorphically onto $\tilde{\mathcal{H}}$; by continuity, this also holds for $t$ sufficiently close to $t_{0}$. This implies that we have a direct sum decomposition $\mathcal{H}=\mathcal{D}_{t} \oplus \mathcal{D}_{t_{0}}^{\perp}$ and the projection $\pi_{t}$ onto $\mathcal{D}_{t}$ is given by:

$$
\pi_{t}=\mathrm{Id}-\left(\left.F(t)\right|_{\mathcal{D}_{t_{0}}^{\perp}}\right)^{-1} \circ F(t) .
$$

Obviously, $t \mapsto \pi_{t}$ is $C^{1}$. For $t$ sufficiently close to $t_{0}$, we define $\alpha(t)$ to be the inverse of the isomorphism:

$$
\left(\pi_{t} \oplus \mathrm{Id}\right): \mathcal{D}_{t_{0}} \oplus \mathcal{D}_{t_{0}}^{\perp} \mapsto \mathcal{D}_{t} \oplus \mathcal{D}_{t_{0}}^{\perp}
$$

Such a map $\alpha$ gives the required local trivialization for the family $\left\{\mathcal{D}_{t}\right\}_{t \in I}$. 
3. Morse-Sturm Systems and the Index Theorem for positive definite metrics.. Motivated by a geometric problem, we introduce a set of data $(g, R, P, S)$ for the Morse-Sturm problem as follows. Let's consider the system of differential equations in $\mathbb{R}^{n}$ :

$$
J^{\prime \prime}(t)=R(t)[J(t)], \quad t \in[0,1]
$$

with initial conditions:

$$
J(0) \in P, \quad J^{\prime}(0)+S[J(0)] \in P^{\perp},
$$

where:

- $g$ is a (fixed) nondegenerate symmetric bilinear form on $\mathbb{R}^{n}$;

- $R:[0,1] \mapsto \mathcal{L}\left(\mathbb{R}^{n}, \mathbb{R}^{n}\right)$ is a continuous map of $g$-symmetric linear maps on $\mathbb{R}^{n}$, i.e., $g(R(t)[x], y)=g(x, R(t)[y])$ for all $x, y \in \mathbb{R}^{n}$;

- $P$ is a subspace of $\mathbb{R}^{n}$ on which $g$ is non degenerate, and $P^{\perp}$ denotes the orthogonal space of $P$ with respect to $g ;{ }^{2}$

- $S: P \mapsto P$ is a $g$-symmetric linear map.

In some of the statements proven in this section, we will assume that $R$ is indeed a map of class $C^{1}$. Nevertheless, some perturbation arguments presented in the next section will allow us to prove our main results in the general case of a continuous map $R$.

A solution for the differential equation (3.1) satisfying the initial conditions (3.2) will be called a $(P, S)$-solution; we denote by $\mathbb{J}$ the set of all $(P, S)$-solutions:

$$
\mathbb{J}=\left\{J:[0,1] \mapsto \mathbb{R}^{n}: J \text { satisfies (3.1) and (3.2) }\right\} \text {. }
$$

Observe that $\mathbb{J}$ is an $n$-dimensional vector space. For all $t \in[0,1]$, we define $\mathbb{J}[t]$ by:

$$
\mathbb{J}[t]=\{J(t): J \in \mathbb{J}\},
$$

and we say that $\left.\left.t_{0} \in\right] 0,1\right]$ is a $(P, S)$-focal instant if there exists a non zero $J \in \mathbb{J}$ such that $J\left(t_{0}\right)=0$. Clearly, this is equivalent to requiring that $\mathbb{J}\left[t_{0}\right] \neq \mathbb{R}^{n}$. The multiplicity $\mu\left(t_{0}\right)$ of a $(P, S)$-focal instant $t_{0}$ is the codimension of $\mathbb{J}\left[t_{0}\right]$ in $\mathbb{R}^{n}$, or equivalently, the dimension of $\mathbb{J}\left[t_{0}\right]^{\perp}$. The signature $\operatorname{sgn}\left(t_{0}\right)$ of $t_{0}$ is defined as the signature of the restriction of the bilinear form $g$ to the space $\mathbb{J}\left[t_{0}\right]^{\perp}$ :

$$
\operatorname{sgn}\left(t_{0}\right)=\operatorname{sgn}\left(\left.g\right|_{\mathbb{J}\left[t_{0}\right]^{\perp}}\right) \text {. }
$$

The $(P, S)$-focal instants coincide with the set of zeroes of the function $r(t)=\operatorname{det}\left(J_{1}(t)\right.$, $\left.J_{2}(t), \ldots, J_{n}(t)\right)$, where $J_{1}, \ldots, J_{n}$ is a basis of $\mathbb{J}$. If $R(t)$ is real analytic, then also $r(t)$ is real analytic on $[0,1]$, hence its zeroes are isolated (observe that $r(t)$ cannot be identically zero, see Proposition 3.1). In [16, Proposition 2.5.1] some sufficient conditions for the discreteness of the $(P, S)$-focal instants are given. More precisely, the following result is proven:

Proposition 3.1. Let $t_{0}$ be $a(P, S)$-focal instant. If $g$ is non degenerate on $\mathbb{J}\left[t_{0}\right]$ (or equivalently on $\mathbb{J}\left[t_{0}\right]^{\perp}$ ) then there are no other $(P, S)$-focal instants in some

\footnotetext{
${ }^{2}$ henceforth, the symbol $\perp$ will mean orthogonality with respect to $g$.
} 
neighborhood of $t_{0}$. Moreover, there are no $(P, S)$-focal instants in some neighborhood of $t_{0}=0$.

A proof of Proposition 3.1 can also be deduced from some results that will be presented in the rest of this section (see Remark 3.6).

An easy calculation shows that, for $J_{1}, J_{2} \in \mathbb{J}$, the following equality holds:

$$
g\left(J_{1}^{\prime}(t), J_{2}(t)\right)=g\left(J_{1}(t), J_{2}^{\prime}(t)\right), \quad \forall t \in[0,1] .
$$

Namely, we use (3.1) to show that the difference $g\left(J_{1}^{\prime}, J_{2}\right)-g\left(J_{1}, J_{2}^{\prime}\right)$ is constant, and (3.2) to see that this constant is zero. Formula (3.6) and an easy dimension counting argument shows that, for $t \in[0,1]$ :

$$
\mathbb{J}[t]^{\perp}=\left\{J^{\prime}(t): J \in \mathbb{J}, J(t)=0\right\} .
$$

Namely, from (3.6) it follows easily the inclusion of the term on the right hand side into $\mathbb{J}[t]^{\perp}$; conversely, it is easy to see that the dimension of the space on the right hand side of $(3.7)$ is equal to $\mu(t)=\operatorname{dim}\left(\mathbb{J}[t]^{\perp}\right)$, which proves (3.7).

Moreover, we introduce the following analytical framework.

Let $H^{1}\left([a, b], \mathbb{R}^{m}\right)$ denote the Sobolev space of all absolutely continuous $\mathbb{R}^{m}$ valued maps on $[a, b]$ with square integrable derivative; $H_{P}^{1}\left([a, b], \mathbb{R}^{m}\right)$ will denote the subspace of $H^{1}\left([a, b], \mathbb{R}^{m}\right)$ consisting of those $V$ such that $V(a) \in P$ and $V(b)=0$. Moreover, $H_{0}^{1}\left([a, b], \mathbb{R}^{m}\right)$ is the subspace of $H^{1}\left([a, b], \mathbb{R}^{m}\right)$ given by the $V$ 's such that $V(a)=V(b)=0$.

For $t \in] 0,1]$, we set $\mathcal{H}_{t}=H_{P}^{1}\left([0, t], \mathbb{R}^{n}\right)$ and $\mathcal{H}=\mathcal{H}_{1} ;$ we define the isomorphisms

$$
\varphi_{t}: \mathcal{H} \mapsto \mathcal{H}_{t}, \quad \text { with } \quad \varphi_{t}(\hat{V})(s)=V(s)=\hat{V}\left(\frac{s}{t}\right), \quad s \in[0, t] .
$$

For each $t \in] 0,1]$, we introduce the index form $I_{t}$ on $\mathcal{H}_{t}$, which is the symmetric bilinear form given by:

$$
I_{t}(V, W)=\int_{0}^{t}\left[g\left(V^{\prime}(s), W^{\prime}(s)\right)+g(R(s)[V(s)], W(s))\right] \mathrm{d} s-g(S[V(0)], W(0)) .
$$

REMARK 3.2. If $g$ is positive definite, then one can consider the following Hilbert space inner product on $\mathcal{H}_{t}$ :

$$
\langle V, W\rangle_{\mathcal{H}_{t}}=\int_{0}^{t} g\left(V^{\prime}(s), W^{\prime}(s)\right) \mathrm{d} s .
$$

The bilinear form $I_{t}$ is written as the sum of $\langle\cdot, \cdot\rangle_{\mathcal{H}_{t}}$ and a bilinear form which is continuous with respect to the $C^{0}$-topology. By the compact embedding of $H^{1}\left([0, t], \mathbb{R}^{n}\right)$ in $C^{0}\left([0, t], \mathbb{R}^{n}\right)$ (see $\left.[6]\right)$, one obtains immediately that $I_{t}$ is of the form $\langle(\operatorname{Id}+K) \cdot, \cdot\rangle_{\mathcal{H}_{t}}$ for some compact operator $K$ on $\mathcal{H}_{t}$.

Finally, for all $t \in] 0,1]$, let $\hat{I}_{t}$ be the symmetric bilinear form on $\mathcal{H}$ obtained by the pull-back of $I_{t}$ by $\varphi_{t}$, namely:

$$
\hat{I}_{t}=I_{t}\left(\varphi_{t} \cdot \varphi_{t}\right)
$$


Explicitly, for $\hat{V}, \hat{W} \in \mathcal{H}$ we have:

$$
\begin{aligned}
& \hat{I}_{t}(\hat{V}, \hat{W}) \\
= & \int_{0}^{t}\left[\frac{1}{t^{2}} g\left(\hat{V}^{\prime}\left(\frac{s}{t}\right), \hat{W}^{\prime}\left(\frac{s}{t}\right)\right)+g\left(R(s)\left[\hat{V}\left(\frac{s}{t}\right)\right], \hat{W}\left(\frac{s}{t}\right)\right)\right] \mathrm{d} s \\
& -g(S[\hat{V}(0)], \hat{W}(0)) .
\end{aligned}
$$

Integration by parts in (3.9) and the Fundamental Lemma of Calculus of Variations show that

$$
\operatorname{Ker}\left(I_{t}\right)=\left\{\left.J\right|_{[0, t]}: J \in \mathbb{J}, J(t)=0\right\}
$$

from (3.7) and (3.12) for each $t \in] 0,1]$ we then get an isomorphism

$$
\begin{aligned}
\psi_{t}: \operatorname{Ker}\left(I_{t}\right) & \longmapsto \mathbb{J}[t]^{\perp} \\
V & \longmapsto V^{\prime}(t) .
\end{aligned}
$$

We set

$$
\mathcal{N}_{t}=\operatorname{Ker}\left(\hat{I}_{t}\right) \subset \mathcal{H}
$$

obviously, $\varphi_{t}$ gives an isomorphism between $\operatorname{Ker}\left(I_{t}\right)$ and $\mathcal{N}_{t}$.

Proposition 3.3. Suppose that $R$ is a map of class $C^{1}$. Then, the map

$$
\text { ]0,1] } \ni t \mapsto \hat{I}_{t} \in \mathrm{B}_{\mathrm{sym}}(\mathcal{H}, \mathbb{R})
$$

is of class $C^{1}$. Moreover, the map ]0,1] $\ni t \mapsto C_{t}=t \cdot \hat{I}_{t}$ has a $C^{1}$-extension to [0,1], with

$$
C_{0}(\hat{V}, \hat{W})=\int_{0}^{1} g\left(\hat{V}^{\prime}(u), \hat{W}^{\prime}(u)\right) \mathrm{d} u, \quad \hat{V}, \hat{W} \in \mathcal{H}
$$

Proof. Substituting $u=s / t$ in (3.11), we get the following expression for $\hat{I}_{t}$ :

$$
\begin{aligned}
\hat{I}_{t}(\hat{V}, \hat{W})= & \int_{0}^{1}\left[\frac{1}{t} g\left(\hat{V}^{\prime}(u), \hat{W}^{\prime}(u)\right)+\operatorname{tg}(R(t u)[\hat{V}(u)], \hat{W}(u))\right] \mathrm{d} u \\
& -g(S[\hat{V}(0)], \hat{W}(0)) .
\end{aligned}
$$

Differentiating (3.16) with respect to $t$ we get:

$$
\begin{aligned}
\frac{\mathrm{d}}{\mathrm{d} t} \hat{I}_{t}(\hat{V}, \hat{W})= & \int_{0}^{1}\left[-\frac{1}{t^{2}} g\left(\hat{V}^{\prime}(u), \hat{W}^{\prime}(u)\right)+g(R(t u)[\hat{V}(u)], \hat{W}(u))\right] \mathrm{d} u \\
& +t \int_{0}^{1} u g\left(R^{\prime}(t u)[\hat{V}(u)], \hat{W}(u)\right) \mathrm{d} u .
\end{aligned}
$$

We now apply Lemma 2.3 to $F(t)=\hat{I}_{t}, G(t)$ is the right hand side of equality (3.17), $E=\mathrm{B}_{\mathrm{sym}}(\mathcal{H}, \mathbb{R}), E_{0}=\mathbb{R}$ and $\Phi=\left\{\phi_{\hat{V}, \hat{W}}: \hat{V}, \hat{W} \in \mathcal{H}\right\}$, where

$$
\phi_{\hat{V}, \hat{W}}(B)=B(\hat{V}, \hat{W}), \quad B \in \mathrm{B}_{\mathrm{sym}}(\mathcal{H}, \mathbb{R}) .
$$


It is easy to check that $G$ is continuous, by the continuity of $R$ and $R^{\prime}$, and clearly $\Phi$ is separating for $E$, which concludes the first part of the proof.

From (3.16) we compute easily:

$$
\begin{aligned}
C_{t}(\hat{V}, \hat{W})= & \int_{0}^{1}\left[g\left(\hat{V}^{\prime}(u), \hat{W}^{\prime}(u)\right)+t^{2} g(R(t u)[\hat{V}(u)], \hat{W}(u))\right] \mathrm{d} u \\
& -t \cdot g(S[\hat{V}(0)], \hat{W}(0)),
\end{aligned}
$$

for all $t \in[0,1]$. Its regularity is established analogously applying Lemma 2.3.

We have the following immediate Corollary:

Corollary 3.4. For $t>0$ small enough, $I_{t}$ is strongly non degenerate on $\mathcal{H}_{t}$. Moreover, if $g$ is positive definite in $\mathbb{R}^{n}$, then $I_{t}$ is positive definite for $t$ small enough.

Proof. For $t>0, I_{t}$ is strongly non degenerate (positive) if and only if $C_{t}$ is strongly non degenerate (positive). From (3.15), $C_{0}$ is strongly non degenerate because $g$ is non degenerate; by continuity, $C_{t}$ is also strongly non degenerate for $t>0$ small enough.

If $g$ is positive definite, then $C_{0}$ is a Hilbert space inner product, and therefore it is positive definite and away from 0 . By continuity, $C_{t}$ is positive definite for $t$ small enough.

We now pass to the study of the signature of $\hat{I}^{\prime}(t)$ on $\mathcal{N}_{t}$. For this, we consider the push-forward of $\hat{I}^{\prime}(t)$ through the isomorphism:

$$
\psi_{t} \circ \varphi_{t}: \mathcal{N}_{t} \longmapsto \mathbb{J}[t]^{\perp}
$$

given by the composition:

$$
\hat{I}^{\prime}(t)\left(\left(\psi_{t} \circ \varphi_{t}\right)^{-1} \cdot,\left(\psi_{t} \circ \varphi_{t}\right)^{-1} \cdot\right),
$$

where the maps $\varphi_{t}$ and $\psi_{t}$ are defined in (3.8) and (3.13).

We have the following:

Proposition 3.5. Suppose that $R$ is a map of class $C^{1}$. For $\left.\left.t \in\right] 0,1\right]$, the isomorphism $\psi_{t} \circ \varphi_{t}$ carries the restriction of $\hat{I}^{\prime}(t)$ to $\mathcal{N}_{t}$ into the restriction of $-g$ to $\mathbb{J}[t]^{\perp}$.

Proof. Let $t \in] 0,1]$ and $\hat{V}, \hat{W} \in \mathcal{N}_{t}$ be fixed; observe that $\hat{V}$ and $\hat{W}$ are maps of class $C^{3}$, because they are affine reparameterizations of solutions to (3.1); they satisfy the following differential equations:

$$
\frac{1}{t^{2}} \hat{V}^{\prime \prime}\left(\frac{s}{t}\right)=R(s)\left[\hat{V}\left(\frac{s}{t}\right)\right], \quad \frac{1}{t^{2}} \hat{W}^{\prime \prime}\left(\frac{s}{t}\right)=R(s)\left[\hat{W}\left(\frac{s}{t}\right)\right], \quad s \in[0, t] .
$$

We differentiate (3.11) with respect to $t$ and, observing that $\hat{V}(1)=\hat{W}(1)=0$, we obtain:

$$
\begin{aligned}
\frac{\mathrm{d}}{\mathrm{d} t} \hat{I}_{t}(\hat{V}, \hat{W})= & \frac{1}{t^{2}} g\left(V^{\prime}(1), W^{\prime}(1)\right)-\int_{0}^{t} \frac{2}{t^{3}} g\left(\hat{V}^{\prime}\left(\frac{s}{t}\right), \hat{W}^{\prime}\left(\frac{s}{t}\right)\right) \mathrm{d} s \\
& -\int_{0}^{t} \frac{s}{t^{4}}\left[g\left(\hat{V}^{\prime \prime}\left(\frac{s}{t}\right), \hat{W}^{\prime}\left(\frac{s}{t}\right)\right)+g\left(\hat{V}^{\prime}\left(\frac{s}{t}\right), \hat{W}^{\prime \prime}\left(\frac{s}{t}\right)\right)\right] \mathrm{d} s \\
& -\int_{0}^{t} \frac{s}{t^{2}}\left[g\left(R(s) \hat{V}^{\prime}\left(\frac{s}{t}\right), \hat{W}\left(\frac{s}{t}\right)\right)+g\left(R(s) \hat{V}\left(\frac{s}{t}\right), \hat{W}^{\prime}\left(\frac{s}{t}\right)\right)\right] \mathrm{d} s .
\end{aligned}
$$


Using (3.19), we eliminate from (3.20) the terms involving the operator $R$, and we get:

$$
\begin{aligned}
\frac{\mathrm{d}}{\mathrm{d} t} \hat{I}_{t}(\hat{V}, \hat{W}) & =\frac{1}{t^{2}} g\left(V^{\prime}(1), W^{\prime}(1)\right)-2 \int_{0}^{t} \frac{\mathrm{d}}{\mathrm{d} s}\left[\frac{s}{t^{3}} g\left(\hat{V}^{\prime}\left(\frac{s}{t}\right), \hat{W}^{\prime}\left(\frac{s}{t}\right)\right)\right] \mathrm{d} s \\
& =-\frac{1}{t^{2}} g(\hat{V}(1), \hat{W}(1))=-g\left(\psi_{t} \circ \varphi_{t}(\hat{V}), \psi_{t} \circ \varphi_{t}(\hat{W})\right) .
\end{aligned}
$$

This concludes the proof.

REMARK 3.6. If $t_{0}$ is a $(P, S)$-focal instant for which $g$ is non degenerate on $\mathbb{J}\left[t_{0}\right]$, then Proposition 2.5 and Proposition 3.5 imply that $\hat{I}_{t}$, and hence $I_{t}$, is non degenerate for $t \neq t_{0}$ sufficiently close to $t_{0}$. Moreover, by Corollary 3.4 there are no $(P, S)$-focal instants near $t=0$. So, we obtain an alternative proof of Proposition 3.1.

As a corollary to Proposition 2.5 and Proposition 3.5, we obtain the classical Morse--Sturm Oscillation Theorem:

Corollary 3.7. Suppose that $R$ is a map of class $C^{1}$. If $g$ is positive definite in $\mathbb{R}^{n}$, then the following equality holds:

$$
n_{-}\left(I_{1}\right)=\sum_{t \in] 0,1[} \mu(t) .
$$

Proof. Let $\left.\left.t_{0} \in\right] 0,1\right]$ be fixed. By Remark $3.2, \hat{I}_{t_{0}}$ is represented by a compact perturbation of the identity map with respect to some suitably chosen Hilbert space inner product on $\mathcal{H}$. By Proposition $3.3, \hat{I}$ is of class $C^{1}$, and we are under the hypotheses of Proposition 2.5. If $t_{0}<1$, applying Corollary 2.8 and Proposition 3.5, we obtain that the integer valued function $i(t)=n_{-}\left(\hat{I}_{t}\right)$ is constant around $t_{0}$ if $t_{0}$ is not a $(P, S)$-focal instant, whereas it has a jump of exactly $\mu\left(t_{0}\right)$ at $t_{0}$ if $t_{0}$ is a $(P, S)$-focal. If $t$ is small enough, by Corollary 3.4 , it is $n_{-}\left(\hat{I}_{t}\right)=0$, and this concludes the proof in the case that $t_{0}=1$ is not $(P, S)$-focal.

Applying Proposition 2.5 to backwards reparameterizations of $\hat{I}_{t}$ (see Remark 2.7), we see that the map $i(t)$ is indeed a left-continuous function on $] 0,1]$, and therefore $n_{-}\left(\hat{I}_{1}\right)=n_{-}\left(\hat{I}_{1-\varepsilon}\right)$ for $\varepsilon>0$ small enough. With this observation the proof is concluded.

4. The Index Theorem for non positive definite metrics. In this section we aim at a generalization of the result of Corollary 3.7 to the case of non positive definite metrics $g$. As we have observed, for a general metric $g$ the left-hand side of the equality (3.22) is infinite; on the other hand, the sum appearing in the right-hand side of (3.22) may lose sense, due the fact that there may be an infinity of focal instants.

For the beginning, we will consider only the case of Morse--Sturm systems having a finite number of $(P, S)$-focal instants. We will see that this assumption holds generically, i.e., for almost all choices of the data $R, P, S$ in (3.1) and (3.2). The conclusion for the general case will be obtained by perturbation arguments, discussed in Section 5. As to the finiteness of the index, we want to consider the restriction of $I_{1}$ to a suitable subspace $\mathcal{K}$ of $\mathcal{H}$ that ought to be small enough to yield finiteness of the index, but large enough to retain the relevant information about the differential problem. Actually, in order to use the techniques of Section 3 to compute the evolution of the index function $i(t)$, we need to determine a whole family $\mathcal{K}_{t}$ of subspaces of $\mathcal{H}_{t}$ with the required properties. 
Having a concrete example in mind, we axiomatize the following set of properties for the family $\mathcal{K}_{t}$.

Definition 4.1. For each $t \in] 0,1]$, let $\mathcal{K}_{t}$ be a closed subspace of $\mathcal{H}_{t}$ and let $\hat{\mathcal{K}}_{t}=\varphi_{t}^{-1}\left(\mathcal{K}_{t}\right)$. The family $\left\{\mathcal{K}_{t}\right\}_{t \in 10,1]}$ is called an admissible family of subspaces for the Morse-Sturm Problem (3.1) and (3.2) if the following conditions are satisfied:

1. the family $\left\{\hat{\mathcal{K}}_{t}\right\}$ admits an extension to $t=0$, denoted by $\hat{\mathcal{K}}_{0}$, which makes it a $C^{1}$-family of closed subspaces on the interval $[0,1]$;

2. for $t \in] 0,1]$, the restriction of the index form $I_{t}$ to $\mathcal{K}_{t}$ is represented by a linear operator which is the sum of a positive self-adjoint isomorphism of $\mathcal{K}_{t}$ and a compact (self-adjoint) operator on $\mathcal{K}_{t}$;

3. the restriction of the bilinear form $C_{0}$ (see formula (3.15)) to $\hat{\mathcal{K}}_{0}$ is non degenerate, and it is represented by the sum of a positive self-adjoint isomorphism and a compact (self-adjoint) operator on $\hat{\mathcal{K}}_{0}$;

4. for $t \in] 0,1]$, the kernel of the restriction of $I_{t}$ to $\mathcal{K}_{t}$ is equal to the kernel of $I_{t}$ in $\mathcal{H}_{t}$ (see formula (3.12)).

The condition 2 of Definition 4.1 implies that, for each $t \in] 0,1]$ there exists a Hilbert space inner product on $\mathcal{K}_{t}$ under which the bilinear form $I_{t}$ is represented by a compact perturbation of the identity map on $\mathcal{K}_{t}$. By condition 3 , the same is true for the bilinear map $C_{0}$ on $\hat{\mathcal{K}}_{0}$. In particular, by the condition 1 and by Proposition 3.3, we are allowed to use the result of Proposition 2.5 and of Corollary 2.8 to the bilinear forms $\hat{I}_{t}$ and $C_{t}$ on $\hat{\mathcal{K}}_{t}$. Observe that the hypothesis 3 of Proposition 2.5 for the family of closed subspaces $\mathcal{K}_{t}$ is satisfied thanks to the axioms 3 and 4 of Definition 4.1.

The axioms satisfied by an admissible family of subspaces for the Morse-Sturm problem constitute the hypotheses of a generalization of Corollary 3.7. Recalling the definition $(3.5)$ of the signature $\operatorname{sgn}(t)$ of a $(P, S)$-focal instant $t$, we prove the following:

TheOREM 4.2. Let $\left\{\mathcal{K}_{t}\right\}_{t \in] 0,1]}$ be an admissible family of subspaces for the MorseSturm problem (3.1) and (3.2), with $R$ of class $C^{1}$, and assume that the restriction of $g$ to $\mathbb{J}[t]$ is non degenerate for all $t \in] 0,1]$. Then, we have the following equality:

$$
n_{-}\left(I_{1} \mid \mathcal{K}_{1}\right)=n_{-}\left(\left.C_{0}\right|_{\hat{\mathcal{K}}_{0}}\right)+\sum_{t \in] 0,1[} \operatorname{sgn}(t)-n_{-}\left(\left.g\right|_{\mathbb{J}[1]^{\perp}}\right) .
$$

Proof. As in the proof of Corollary 3.7, we study the evolution of the function $i(t)=n_{-}\left(\left.\hat{I}_{t}\right|_{\hat{\mathcal{K}}_{t}}\right)$ when $t$ runs from 0 to 1 ; observe that $i(1)=n_{-}\left(\left.\hat{I}_{1}\right|_{\hat{\mathcal{K}}_{1}}\right)=n_{-}\left(I_{1} \mid \mathcal{K}_{1}\right)$. Observe that, by the axiom 4 of Definition 4.1 , the $(P, S)$-focal instants coincide with the instants $t$ where $\hat{I}_{t}$ is degenerate on $\hat{\mathcal{K}}_{t}$.

By Proposition 3.1 (see also Remark 3.6), there is only a finite number of $(P, S)$ focal instants, hence, by Remark 2.7, $i$ is piecewise constant on ]0,1]. Namely, $i$ is constant on any interval that does not contain $(P, S)$-focal instants.

Since $n_{-}\left(\left.C_{t}\right|_{\hat{\mathcal{K}}_{t}}\right)=n_{-}\left(\left.\hat{I}_{t}\right|_{\hat{\mathcal{K}}_{t}}\right)$ for $t>0$, by the non degeneracy of $C_{0}$ on $\hat{\mathcal{K}}_{0}$ and Remark 2.7, $i(t)=n_{-}\left(\left.C_{0}\right|_{\hat{\mathcal{K}}_{0}}\right)$ for $t>0$ sufficiently small.

When $t$ passes through a $(P, S)$-focal instant $\left.t_{0} \in\right] 0,1[$, by Corollary 2.8 and by Proposition 3.5 the jump of the function $i$ is equal to the signature $\operatorname{sgn}\left(t_{0}\right)$.

Finally, applying Proposition 2.5 to a backwards reparameterization of $\hat{I}_{t}$ around $t_{0}=1$ (see Remark 2.7), by Proposition 3.5 for $t<1$ sufficiently close to 1 we have $i(t)-i(1)=n_{-}\left(\left.g\right|_{J[1]} ^{\perp}\right)$, which concludes the proof. 
We have observed in the proof of Corollary 3.7 that the index function $i(t)$ is left-continuous under the positivity assumption for $g$. We emphasize that, as it was clear in the above proof, this property fails when $g$ is non positive. As a consequence of this lack of continuity, when comparing with the Riemannian Index Theorem, in the right hand side of equality (4.1) we get the extra term $n_{-}\left(\left.g\right|_{\mathbb{J}[1]^{\perp}}\right)$ which is non zero when $t_{0}=1$ is $(P, S)$-focal.

Another remarkable phenomenon that appears in the case of non positive definite metrics is the presence of the term $n_{-}\left(\left.C_{0}\right|_{\hat{\mathcal{K}}_{0}}\right)$ in the equality $(4.1)$, which is the initial value of the index function $i(t)$. As we saw in the proof of Corollary 3.7, for positive definite metrics, such initial value is zero.

We now present a concrete example of the above situation. We will assume throughout the rest of this section that $n_{-}(g)=1$ and that the differential equation (3.1) admits a solution $Y:[0,1] \mapsto \mathbb{R}^{n}$ with the property that $g(Y, Y)<0$ on $[0,1]$ :

$$
Y^{\prime \prime}=R Y, \text { and } g(Y, Y)<0 .
$$

We fix one such solution $Y$ and we consider the following one-parameter family of positive definite inner products in $\mathbb{R}^{n}$ :

$$
g_{t}^{(\mathrm{r})}(v, w)=g(v, w)-2 \frac{g(v, Y(t)) g(w, Y(t))}{g(Y(t), Y(t))}, \quad \forall t \in[0,1], v, w \in \mathbb{R}^{n} .
$$

Observe that, for all $t \in[0,1], g_{t}^{(\mathrm{r})}(v, w)$ coincides with $g(v, w)$ if either $v$ or $w$ is orthogonal to $Y(t)$, and $g_{t}^{(\mathbf{r})}(Y(t), Y(t))=-g(Y(t), Y(t))$. The formula that gives $g$ in terms of $g_{t}^{(\mathrm{r})}$ is similar:

$$
g(v, w)=g_{t}^{(\mathrm{r})}(v, w)-2 \frac{g_{t}^{(\mathrm{r})}(v, Y(t)) g_{t}^{(\mathrm{r})}(w, Y(t))}{g_{t}^{(\mathrm{r})}(Y(t), Y(t))} .
$$

For all $t \in] 0,1]$, we consider the following subspace of $\mathcal{H}_{t}$ :

$$
\mathcal{K}_{t}=\left\{V \in \mathcal{H}_{t}: g\left(V^{\prime}, Y\right)-g\left(V, Y^{\prime}\right) \equiv C_{V} \text { (constant) }\right\} .
$$

We claim that $\mathcal{K}_{t}$ is an admissible family of subspaces for the Morse-Sturm Problem (3.1) and (3.2), and we take the rest of this section to prove the claim.

As in Definition 4.1, for $t \in] 0,1]$ we set $\hat{\mathcal{K}}_{t}=\varphi_{t}^{-1}\left(\mathcal{K}_{t}\right)$; explicitly, we have:

$$
\left.\hat{\mathcal{K}}_{t}=\left\{\hat{V} \in \mathcal{H}: g\left(\hat{V}^{\prime}(u), \hat{Y}_{t}(u)\right)-g\left(\hat{V}(u), \hat{Y}_{t}^{\prime}(u)\right)\right) \equiv \text { const. }\right\},
$$

where $\hat{Y}_{t}(u)=Y(t \cdot u)$ for $u \in[0,1]$. We observe that formula (4.6) makes sense also for $t=0$, where $\hat{Y}_{0}$ is the constant vector $Y(0)$ :

$$
\hat{\mathcal{K}}_{0}=\left\{\hat{V} \in \mathcal{H}: g\left(\hat{V}^{\prime}(u), Y(0)\right) \equiv \text { const. }\right\} .
$$

Let $\tilde{\mathcal{H}}$ denote the Hilbert space given by the quotient $L^{2}([0,1], \mathbb{R}) / \mathfrak{C}$, where $\mathfrak{C}$ denotes the subspace of constant functions. For $t \in[0,1], \hat{\mathcal{K}}_{t}$ is the kernel of the bounded linear map $F_{t}: \mathcal{H} \mapsto \tilde{\mathcal{H}}$ given by:

$$
\begin{aligned}
F_{t}(\hat{V})(u) & =g\left(\hat{V}^{\prime}(u), \hat{Y}_{t}(u)\right)-g\left(\hat{V}(u), \hat{Y}_{t}^{\prime}(u)\right)+\mathfrak{C} \\
& =g\left(\hat{V}^{\prime}(u), Y(t u)\right)-t \cdot g\left(\hat{V}(u), Y^{\prime}(t u)\right)+\mathfrak{C} .
\end{aligned}
$$


LEMMA 4.3. The map $[0,1] \ni t \mapsto F_{t} \in \mathcal{L}(\mathcal{H}, \widetilde{\mathcal{H}})$ is of class $C^{1}$.

Proof. We formally differentiate (4.8), obtaining:

$$
F_{t}^{\prime}(V)(u)=u g\left(\hat{V}^{\prime}(u), Y^{\prime}(t u)\right)-g\left(\hat{V}(u), Y^{\prime}(t u)\right)-t u g\left(\hat{V}(u), Y^{\prime \prime}(t u)\right)+\mathfrak{C} .
$$

Using the fact that $Y$ is of class $C^{2}$, it is easily seen that formula (4.9) defines a continuous curve in $\mathcal{L}(\mathcal{H}, \widetilde{\mathcal{H}})$. We now use Lemma 2.3 by considering $\Phi$ to be the set of evaluations at fixed vectors $\hat{V} \in \mathcal{H}$; the conclusion will follow once we prove that the map $t \mapsto F_{t}(\hat{V}) \in \widetilde{\mathcal{H}}$ is of class $C^{1}$ for all $\hat{V} \in \mathcal{H}$, and that its derivative is given by (4.9).

Let $C^{1}\left([0,1], \mathbb{R}^{n}\right)$ be the Banach space of $\mathbb{R}^{n}$-valued $C^{1}$-maps on $[0,1]$; we define the following bounded linear operator $\sigma: C^{1}\left([0,1], \mathbb{R}^{n}\right) \mapsto \tilde{\mathcal{H}}$ by:

$$
\sigma(\mathcal{Y})(u)=g\left(\hat{V}^{\prime}(u), \mathcal{Y}(u)\right)-g\left(\hat{V}(u), \mathcal{Y}^{\prime}(u)\right) .
$$

We observe that the map $t \mapsto F_{t}(\hat{V})$ is given by the composition of $\sigma$ and the map

$$
t \mapsto \hat{Y}_{t} \in C^{1}\left([0,1], \mathbb{R}^{n}\right) .
$$

It remains to show that the map (4.11) is of class $C^{1}$. This is again an easy consequence of Lemma 2.3, where $\Phi$ is the set of evaluations at fixed instants $u \in[0,1]$.

The next step towards our goal is to prove the surjectivity of $F_{t}$. We introduce the subspaces $\mathcal{S}_{t} \subset \mathcal{H}_{t}$ and $\hat{\mathcal{S}}_{t} \subset \mathcal{H}$ :

$$
\begin{aligned}
& \left.\left.\mathcal{S}_{t}=\left\{\left.f \cdot Y\right|_{[0, t]}: f \in H_{0}^{1}([0, t], \mathbb{R})\right\}, \quad t \in\right] 0,1\right], \\
& \hat{\mathcal{S}}_{t}=\left\{\hat{f} \cdot \hat{Y}_{t}: \hat{f} \in H_{0}^{1}([0,1], \mathbb{R})\right\}, \quad t \in[0,1] .
\end{aligned}
$$

Observe that, for $t \in] 0,1], \mathcal{S}_{t}=\varphi_{t}\left(\hat{\mathcal{S}}_{t}\right)$. We show now that $F_{t}\left(\hat{\mathcal{S}}_{t}\right)=\tilde{\mathcal{H}}$ :

Lemma 4.4. For all $t \in[0,1]$, the restriction of $F_{t}$ to $\hat{\mathcal{S}}_{t}$ is surjective.

Proof. For $\hat{f} \in H_{0}^{1}([0,1], \mathbb{R})$, we compute :

$$
F_{t}\left(\hat{f} \cdot \hat{Y}_{t}\right)=\hat{f}^{\prime} \cdot g\left(\hat{Y}_{t}, \hat{Y}_{t}\right)+\mathfrak{C}
$$

Hence, for the proof we need to show that, given $h \in L^{2}([0,1], \mathbb{R})$ there exists $c \in \mathbb{R}$ and $\hat{f} \in H_{0}^{1}([0,1], \mathbb{R})$ such that the following differential equation is satisfied:

$$
\hat{f}^{\prime}=\frac{h+c}{g\left(\hat{Y}_{t}, \hat{Y}_{t}\right)}
$$

It suffices to take:

$$
c=-\left(\int_{0}^{1} \frac{\mathrm{d} r}{g\left(\hat{Y}_{t}, \hat{Y}_{t}\right)}\right)^{-1} \int_{0}^{1} \frac{h}{g\left(\hat{Y}_{t}, \hat{Y}_{t}\right)} \mathrm{d} r, \quad \text { and } \quad \hat{f}(u)=\int_{0}^{u} \frac{h+c}{g\left(\hat{Y}_{t}, \hat{Y}_{t}\right)} \mathrm{d} r .
$$

Observe that the above formulas make sense because $g\left(\hat{Y}_{t}, \hat{Y}_{t}\right)<0$.

Corollary 4.5. $\left\{\hat{\mathcal{K}}_{t}\right\}_{t \in[0,1]}$ is a $C^{1}$-family of closed subspaces of $\mathcal{H}$. 
Proof. It follows directly from Lemma 2.9, Lemma 4.3 and Lemma 4.4.

Corollary 4.6. For $t \in] 0,1], \mathcal{H}_{t}=\mathcal{K}_{t}+\mathcal{S}_{t}$; moreover, $\mathcal{H}=\hat{\mathcal{K}}_{0}+\hat{\mathcal{S}}_{0}$.

Proof. By Lemma 4.4, an easy linear algebra argument shows that, for $t \in[0,1]$, $\mathcal{H}=\hat{\mathcal{K}}_{t}+\hat{\mathcal{S}}_{t}$. For $\left.\left.t \in\right] 0,1\right]$ we apply the isomorphism $\varphi_{t}$ and we get the conclusion. $\square$

Although we will not need it, we emphasize that the sums in the statement of Corollary 4.6 are direct. As a matter of facts, we now prove that the above sums are orthogonal with respect to the bilinear forms $I_{t}$ and $C_{0}$, respectively.

Lemma 4.7. For all $t \in] 0,1]$, the spaces $\mathcal{K}_{t}$ and $\mathcal{S}_{t}$ are orthogonal with respect to the bilinear form $I_{t}$; moreover, the spaces $\hat{\mathcal{K}}_{0}$ and $\hat{\mathcal{S}}_{0}$ are orthogonal with respect to $C_{0}$.

Proof. Let $V \in \mathcal{K}_{t}$ and $f \cdot Y \in \mathcal{S}_{t}$ be fixed, with $f(0)=f(t)=0$. From (3.9), (4.2) and (4.5), we compute using integration by parts as follows:

$$
\begin{aligned}
I_{t}(V, f Y) & =\int_{0}^{t}\left[f^{\prime} g\left(V^{\prime}, Y\right)+f g\left(V^{\prime}, Y^{\prime}\right)+f g(R V, Y)\right] \mathrm{d} s \\
& =\int_{0}^{t}\left[f^{\prime} C_{V}+f^{\prime} g\left(V, Y^{\prime}\right)+f g\left(V^{\prime}, Y^{\prime}\right)+f g(R V, Y)\right] \mathrm{d} s \\
& =\int_{0}^{t}\left[-f g\left(V^{\prime}, Y^{\prime}\right)-f g\left(V, Y^{\prime \prime}\right)+f g\left(V^{\prime}, Y^{\prime}\right)+f g(V, R Y)\right] \mathrm{d} s \\
& =0 .
\end{aligned}
$$

Similarly, if $\hat{V} \in \hat{\mathcal{K}}_{0}$ and $f \cdot Y(0) \in \hat{\mathcal{S}}_{0}$ are fixed, $f(0)=f(1)=0$, since $g\left(\hat{V}^{\prime}, Y(0)\right)$ is constant, from (3.15) we have:

$$
C_{0}(\hat{V}, f \cdot Y(0))=\int_{0}^{1} f^{\prime} g\left(\hat{V}^{\prime}, Y(0)\right) \mathrm{d} u=0,
$$

which concludes the proof.

CoROllary 4.8. For all $t>0$, the kernel of the restriction of $I_{t}$ to $\mathcal{K}_{t}$ equals the kernel of $I_{t}$ in $\mathcal{H}_{t}$ (see formula (3.12)); moreover, $C_{0}$ is non degenerate in $\hat{\mathcal{K}}_{0}$.

Proof. Let $t \in] 0,1]$ be fixed. From (3.6), (3.12) and (4.5) it follows immediately that $\operatorname{Ker}\left(I_{t}\right) \subset \mathcal{K}_{t}$, hence $\operatorname{Ker}\left(I_{t}\right) \subset \operatorname{Ker}\left(I_{t} \mid \mathcal{K}_{t}\right)$.

For the opposite inclusion, observe that, if $V \in \operatorname{Ker}\left(I_{t} \mid \mathcal{K}_{t}\right)$, then $I_{t}(V, W)=0$ for all $W \in \mathcal{K}_{t}$, and, by Lemma 4.7, also $I_{t}(V, W)=0$ for all $W \in \mathcal{S}_{t}$. By Corollary 4.6 it then follows that $I_{t}(V, W)=0$ for all $W \in \mathcal{H}_{t}$, proving that $\operatorname{Ker}\left(I_{t}\right) \supset \operatorname{Ker}\left(\left.I_{t}\right|_{\mathcal{K}_{t}}\right)$.

Similarly, $\operatorname{Ker}\left(C_{0}\right)=\operatorname{Ker}\left(\left.C_{0}\right|_{\hat{\mathcal{K}}_{0}}\right)$. Since $g$ is non degenerate, from (3.15) it is easy to see that $C_{0}$ is non degenerate in $\mathcal{H}$, which proves that $C_{0}$ is non degenerate in $\hat{\mathcal{K}}_{0}$.

We now look at the representation of the bilinear forms $I_{t}$ and $C_{0}$ as self-adjoint operators. We start with the following general observation.

If $B: H^{1}\left([0,1], \mathbb{R}^{n}\right) \times H^{1}\left([0,1], \mathbb{R}^{n}\right) \mapsto \mathbb{R}$ is a bilinear form obtained by the restriction of a continuous bilinear form on $C^{0}\left([0,1], \mathbb{R}^{n}\right) \times C^{0}\left([0,1], \mathbb{R}^{n}\right)$, then, since the inclusion $H^{1} \mapsto C^{0}$ is compact, it follows that $B$ is represented by a compact operator on $H^{1}\left([0,1], \mathbb{R}^{n}\right)$.

We can now prove the following: 
Proposition 4.9. For all $t>0, I_{t}$ is represented by a self-adjoint bounded linear operator on $\mathcal{K}_{t}$ which is of the form $L+K$, where $L$ is a positive isomorphism of $\mathcal{K}_{t}$ and $K$ is compact. Also, the restriction of $C_{0}$ to $\hat{\mathcal{K}}_{0}$ is represented by a compact perturbation of the identity map of $\hat{\mathcal{K}}_{0}$. follows:

Proof. Let $t \in] 0,1]$ be fixed; from (3.9), (4.4) and (4.5) we write $I_{t}$ on $\mathcal{K}_{t}$ as

$$
\begin{aligned}
I_{t}(V, W)= & \int_{0}^{t} g_{s}^{(\mathrm{r})}\left(V^{\prime}(s), W^{\prime}(s)\right) \mathrm{d} s \\
& +2 \int_{0}^{t} \frac{\left[C_{V}+g\left(V(s), Y^{\prime}(s)\right)\right]\left[C_{W}+g\left(W(s), Y^{\prime \prime}(s)\right)\right]}{g(Y(s), Y(s))} \mathrm{d} s \\
& +\int_{0}^{t} g(R(s)[V(s)], W(s)) \mathrm{d} s-g(S[V(0)], W(0)) .
\end{aligned}
$$

Now, the bilinear form on $\mathcal{K}_{t}$ given by the first integral in (4.14) is a Hilbert space inner product on $\mathcal{K}_{t}$, and therefore it is represented by the identity operator on $\mathcal{K}_{t}$.

We now observe that the bounded linear operator

$$
V \mapsto C_{V}=\frac{1}{t} \int_{0}^{t}\left[g\left(V^{\prime}, Y\right)-g\left(V, Y^{\prime}\right)\right] \mathrm{d} s
$$

from $H^{1}\left([0, t], \mathbb{R}^{n}\right)$ to $\mathbb{R}$ has a continuous extension to $C^{0}\left([0,1], \mathbb{R}^{n}\right)$. Namely:

$$
C_{V}=\frac{1}{t} \int_{0}^{t}\left[g\left(V^{\prime}, Y\right)-g\left(V, Y^{\prime}\right)\right] \mathrm{d} s=\frac{1}{t}\left[\left.g(V, Y)\right|_{0} ^{t}-2 \int_{0}^{t} g\left(V, Y^{\prime}\right) \mathrm{d} s\right],
$$

and the latter expression is clearly continuous with respect to the uniform topology. It follows that the bilinear form on $\mathcal{K}_{t}$ given by the second integral of formula (4.14) has a continuous extension to $C^{0}\left([0,1], \mathbb{R}^{n}\right)$, and we have observed that this implies that it is represented by a compact operator on $\mathcal{K}_{t}$. The terms in the last line of formula (4.14) are also continuous in the $C^{0}$-topology, and again the corresponding bilinear form is represented by a compact operator on $\mathcal{K}_{t}$, which proves the first part of the Proposition.

As to the bilinear form $C_{0}$ on $\hat{\mathcal{K}}_{0}$, observe that, by definition of $\hat{\mathcal{K}}_{0}$ (see formula (4.7)), if $\hat{V} \in \hat{\mathcal{K}}_{0}$ then the quantity $g_{0}^{(\mathrm{r})}\left(\hat{V}^{\prime}, Y(0)\right)=-g\left(\hat{V}^{\prime}, Y(0)\right)$ is constant, and thus:

$$
g_{0}^{(\mathrm{r})}\left(\hat{V}^{\prime}, Y(0)\right)=\int_{0}^{1} g_{0}^{(\mathrm{r})}\left(\hat{V}^{\prime}, Y(0)\right) \mathrm{d} u=-g_{0}^{(\mathrm{r})}(\hat{V}(0), Y(0)) .
$$

Then, for $\hat{V}, \hat{W} \in \hat{\mathcal{K}}_{0}$, it is:

$$
C_{0}(\hat{V}, \hat{W})=\int_{0}^{1} g_{0}^{(\mathrm{r})}\left(\hat{V}^{\prime}(u), \hat{W}^{\prime}(u)\right) \mathrm{d} u-2 \frac{g_{0}^{(\mathrm{r})}(\hat{V}(0), Y(0)) g_{0}^{(\mathrm{r})}(\hat{W}(0), Y(0))}{g_{0}^{(\mathrm{r})}(Y(0), Y(0))}
$$

Again, the integral in the above formula is a Hilbert space inner product in $\hat{\mathcal{K}}_{0}$, and the last term is continuous in the $C^{0}$-topology, which proves that $C_{0}$ is represented by a compact perturbation of a positive isomorphism of $\hat{\mathcal{K}}_{0}$.

Proposition 4.10. The index of $C_{0}$ in $\hat{\mathcal{K}}_{0}$ is equal to the index of the restriction of $g$ to the subspace $P$ :

$$
n_{-}\left(\left.C_{0}\right|_{\hat{\mathcal{K}}_{0}}\right)=n_{-}\left(\left.g\right|_{P}\right)
$$


Proof. Let $P=P_{+} \oplus P_{-}$be a direct sum decomposition of $P$, with $\left.g\right|_{P_{+}}$positive definite and $\left.g\right|_{P_{-}}$negative definite (recall that $g$ is non degenerate on $P$ ). Then, it is easy to see that we have a direct sum decomposition $\hat{\mathcal{K}}_{0}=\hat{\mathcal{K}}_{+} \oplus \hat{\mathcal{K}}_{-}$, where:

$$
\hat{\mathcal{K}}_{+}=\left\{\hat{V} \in \hat{\mathcal{K}}_{0}: \hat{V}(0) \in P_{+}\right\}
$$

and

$$
\hat{\mathcal{K}}_{-}=\left\{\hat{V}:[0,1] \mapsto \mathbb{R}^{n} \text { affine function } \mid \hat{V}(0) \in P_{-}, \hat{V}(1)=0\right\} .
$$

Clearly, $\operatorname{dim}\left(\hat{\mathcal{K}}_{-}\right)=\operatorname{dim}\left(P_{-}\right)=n_{-}\left(\left.g\right|_{P}\right) ;$ to conclude the proof, it suffices to show that $C_{0}$ is positive semi-definite on $\hat{\mathcal{K}}_{+}$and negative definite in $\hat{\mathcal{K}}_{-}$.

If $\hat{V} \in \hat{\mathcal{K}}_{-}, \hat{V} \neq 0$, then $\hat{V}(u)=v_{0}(u-1)$ for some $v_{0} \in P_{-}, v_{0} \neq 0$, and for all $u \in[0,1]$; then, from (3.15), we have:

$$
C_{0}(\hat{V}, \hat{V})=\int_{0}^{1} g\left(\hat{V}^{\prime}, \hat{V}^{\prime}\right) \mathrm{d} u=g\left(v_{0}, v_{0}\right)<0
$$

If $\hat{V} \in \hat{\mathcal{K}}_{+}$, then, by (4.16), we have:

$$
C_{0}(\hat{V}, \hat{V})=\int_{0}^{1} g_{0}^{(\mathrm{r})}\left(\hat{V}^{\prime}, \hat{V}^{\prime}\right) \mathrm{d} u-2 \frac{g_{0}^{(\mathrm{r})}(\hat{V}(0), Y(0))^{2}}{g_{0}^{(\mathrm{r})}(Y(0), Y(0))}
$$

Since $\hat{V}(1)=0$ ad the function $v \mapsto g_{0}^{(\mathrm{r})}(v, v)$ is convex in $\mathbb{R}^{n}$, we use the Jensen's inequality to prove the following:

$$
g_{0}^{(\mathrm{r})}(\hat{V}(0), \hat{V}(0))=g_{0}^{(\mathrm{r})}\left(\int_{0}^{1} \hat{V}^{\prime} \mathrm{d} u, \int_{0}^{1} \hat{V}^{\prime} \mathrm{d} u\right) \leq \int_{0}^{1} g_{0}^{(\mathrm{r})}\left(\hat{V}^{\prime}(u), \hat{V}^{\prime}(u)\right) \mathrm{d} u .
$$

Finally, from (4.20) and (4.21) we obtain:

$$
C_{0}(\hat{V}, \hat{V}) \geq g_{0}^{(\mathrm{r})}(\hat{V}(0), \hat{V}(0))-2 \frac{g_{0}^{(\mathrm{r})}(\hat{V}(0), Y(0))^{2}}{g_{0}^{(\mathrm{r})}(Y(0), Y(0))}=g(\hat{V}(0), \hat{V}(0)) \geq 0
$$

which concludes the proof.

We summarize the above results in the next theorem:

THEOREM 4.11. Let $g$ be a nondegenerate symmetric bilinear form on $\mathbb{R}^{n}$ with $n_{-}(g)=1, R:[0,1] \mapsto \mathcal{L}\left(\mathbb{R}^{n}\right)$ be a $C^{1}$-map of $g$-symmetric linear operators on $\mathbb{R}^{n}$, $P$ a g-nondegenerate subspace of $\mathbb{R}^{n}$ and $S: P \mapsto P$ be a $g$-symmetric linear map on $P$. Suppose that the differential equation $V^{\prime \prime}=R V$ admits a solution $Y$ satisfying $g(Y, Y)<0$ on $[0,1]$. Let $\mathcal{K}$ be the subspace of $H_{P}^{1}\left([0,1], \mathbb{R}^{n}\right)$ consisting of those $V$ such that $g\left(V^{\prime}, Y\right)-g\left(V, Y^{\prime}\right)$ is constant on $[0,1]$; assume that $g$ is non degenerate on each $\mathbb{J}[t]$. Then

$$
n_{-}\left(\left.I_{1}\right|_{\mathcal{K}}\right)=n_{-}\left(\left.g\right|_{P}\right)+\sum_{t \in] 0,1[} \operatorname{sgn}(t)-n_{-}\left(\left.g\right|_{\mathbb{J}[1]^{\perp}}\right)
$$

where the objects $I_{1}$ and $\mathbb{J}[t]$ are defined in (3.9) and (3.4). 
5. On the Nondegeneracy Assumption. The Maslov Index... In this section we will discuss the nondegeneracy assumption for the restriction of the bilinear form $g$ on the spaces $\mathbb{J}[t]$ defined in (3.4), and which is essential for the proof of Theorem 4.2.

As we have observed, this assumption guarantees that the set of $(P, S)$-focal instants is discrete (Proposition 3.1); however, it is important to observe that, even when the number of $(P, S)$-focal instants is finite, such assumption cannot be removed from the statement of Theorem 4.2 (see [16, Section 7]).

A natural substitute for the term $\sum_{t \in] 0,1[} \operatorname{sgn}(t)$ appearing in formula (4.1) in the case that $g$ is possibly degenerate on some $\mathbb{J}[t]$ is the so called Maslov index of the differential problem (3.1) and (3.2), denoted by $\mathrm{i}_{\mathrm{M}}(g, R, P, S)$ (see [12, 16] for details). The Maslov index $\mathrm{i}_{\mathrm{M}}(g, R, P, S)$ is defined whenever $t_{0}=1$ is not a $(P, S)$-focal instant. It is an integer number computed as the intersection number of a continuous curve with a subvariety of codimension one of the Lagrangian Grassmannian of a symplectic space.

For the reader's convenience, we sketch briefly the formal definition of $i_{M}$; the proofs and further details on our approach may be found, in [16]. Consider the differential problem in $\mathbb{R}^{n}$ given by (3.1) and (3.2). Using the bilinear form $g$, one considers the symplectic form $\omega$ in $\mathbb{R}^{2 n}$ given by:

$$
\omega\left(\left(v_{1}, v_{2}\right),\left(w_{1}, w_{2}\right)\right)=g\left(v_{1}, w_{2}\right)-g\left(v_{2}, w_{1}\right) .
$$

It is an easy observation that, if $V$ and $W$ are solutions of (3.1), then the quantity $\omega\left(\left(V(t), V^{\prime}(t)\right),\left(W(t), W^{\prime}(t)\right)\right.$ is constant in $[a, b]$; moreover, if $V$ and $W$ are in $\mathbb{J}$, then this constant is null (see formula (3.6)). A subspace $L$ of $\mathbb{R}^{2 n}$ is said to be isotropic with respect to $\omega$ if $\omega$ is null on $L \times L$; the space

$$
L=\left\{\left(v_{1}, v_{2}\right) \in \mathbb{R}^{2 n}: v_{1} \in P, v_{2}+S\left[v_{1}\right] \in P^{\perp}\right\}
$$

is a Lagrangian subspace of the symplectic space $\left(\mathbb{R}^{2 n}, \omega\right)$, which is a maximal isotropic subspace of $\mathbb{R}^{2 n}$ (necessarily $n$-dimensional). The set $\Lambda$ consisting of all the Lagrangian subspaces of the symplectic space $\left(\mathbb{R}^{2 n}, \omega\right)$ is a compact, connected, analytic embedded submanifold of the Grassmannian $G_{n}\left(\mathbb{R}^{2 n}\right)$, called the Lagrangian Grassmannian of $\left(\mathbb{R}^{2 n}, \omega\right)$.

By what has been observed, for all $t \in[a, b]$, the subspace of $\mathbb{R}^{2 n}$ given by:

$$
L(t)=\left\{\left(V(t), V^{\prime}(t)\right): V \in \mathbb{J}\right\}
$$

is Lagrangian, hence the differential problem (3.1) and (3.2) defines a continuous curve in $\Lambda$. Considering the Lagrangian subspace of $\mathbb{R}^{2 n}$ :

$$
L_{0}=\{0\} \oplus \mathbb{R}^{n},
$$

it is an easy observation that an instant $\left.\left.t_{0} \in\right] a, b\right]$ is $P$-focal if and only if $L\left(t_{0}\right) \cap L_{0} \neq$ $\{0\}$, i.e., if and only if $L\left(t_{0}\right)$ and $L_{0}$ are transversal. One then considers the subset $\Lambda_{0} \subset \Lambda$ consisting of those Lagrangians that are transversal to $L_{0} ; \Lambda_{0}$ is a dense open subset of $\Lambda$ which is contractible. The first relative homology group with integer coefficients $H_{1}\left(\Lambda, \Lambda_{0} ; \mathbb{Z}\right)$ is computed in [16] as:

$$
H_{1}\left(\Lambda, \Lambda_{0} ; \mathbb{Z}\right) \simeq \mathbb{Z}
$$


The continuous curve $L(t)$ in $\Lambda$ defined by our differential problem does not define a homology class in $H_{1}\left(\Lambda, \Lambda_{0} ; \mathbb{Z}\right)$, because its initial point is never in $\Lambda_{0}$; moreover, its final point is in $\Lambda_{0}$ precisely when $t_{0}=b$ is not a $P$-focal point. Let's assume that $t_{0}=b$ is not a $P$-focal point; by Proposition 3.1, if we consider the restriction $L_{\varepsilon}$ of the curve $L(t)$ to an interval of the form $[a+\varepsilon, b]$, with $\varepsilon>0$ small enough, then we have a well defined continuous curve in $\Lambda$ with endpoints in $\Lambda_{0}$. The relative homology class of this curve is easily seen not to depend on the choice of the small $\varepsilon$. The Maslov index $\mathrm{i}_{\mathrm{M}}(g, R, P, S)$ is defined to be the relative homology class of $L_{\varepsilon}$ in $H_{1}\left(\Lambda, \Lambda_{0} ; \mathbb{Z}\right)$.

Such index equals the sum $\sum_{t \in] 0,1[} \operatorname{sgn}(t)$ when the non degeneracy assumption for $g$ is satisfied ([16, Theorem 5.1.2]). Moreover, the essential property of $\mathrm{i}_{\mathrm{M}}$ is that, since it is a topological invariant, it is stable by $C^{0}$-small perturbations of the data $(g, R, P, S)([16$, Theorem 5.2.1]). As an immediate application of the uniform stability of $\mathrm{i}_{\mathrm{M}}$, we obtain immediately that the result of Theorem 4.11 can be extended to the case that $R$ is only continuous, provided that the instant $t_{0}=1$ is not $(P, S)$-focal, by replacing the term $\sum_{t \in] 0,1[} \operatorname{sgn}(t)$ in (4.22) with the Maslov index $\mathrm{i}_{\mathrm{M}}(g, R, P, S)$.

Using a similar perturbation argument, we now want to push the result of Theorem 4.11 beyond the assumption of non degeneracy for $g$. To this aim, we argue as follows.

Let's assume that a set of data $(g, R, P, S)$ is given in $\mathbb{R}^{n}$, with $n_{-}(g)=1$, and suppose that the following assumptions are satisfied:

(a) $g$ is non degenerate on $P$;

(b) the differential equation $V^{\prime \prime}=R V$ admits a solution $Y$ satisfying $g(Y, Y)<0$ in $[0,1]$

(c) the instant $t_{0}=1$ is not $(P, S)$-focal.

If $g^{\prime}$ is a symmetric bilinear form on $\mathbb{R}^{n}$ which is sufficiently close to $g$ and $P^{\prime}$ is a subspace of $\mathbb{R}^{n}$ sufficiently close to $P$ (in the sense of the Grassmannian of subspaces of $\mathbb{R}^{n}$ ), then clearly $n_{-}\left(g^{\prime}\right)=1$ and $g^{\prime}$ is non degenerate on $P^{\prime}$. So, the assumption (a) above is stable by small perturbations.

Moreover, standard results on the continuous dependence from the data for ordinary differential equations guarantee that also the assumptions (b) and (c) above are stable by uniformly small perturbations of the objects $g, R, P$ and $S$.

Finally, to complete the argument, we need to prove that it is possible to produce arbitrarily $C^{0}$-small perturbations of the data $(g, R, P, S)$ for which the restriction of $g$ to the spaces $\mathbb{J}[t]$ is non degenerate for all $t \in] 0,1]$. It is easy to prove that such perturbations of the Morse-Sturm problem (3.1) and (3.2) exist in the more general class of linearized Hamiltonian systems, where some of the results of this paper and of [16] still hold in a more general form. In this class, the set of systems for which the non degeneracy assumption is $C^{0}$-dense. Since both the Morse index and the Maslov index are stable by uniformly small perturbations (see [16]), we obtain the following extension of Theorem 4.11:

TheOREM 5.1. Let $(g, R, P, S)$ be a set of data for the Morse-Sturm problem (3.1) and (3.2). Suppose that the following assumptions are satisfied:

- $n_{-}(g)=1$;

- $R$ is continuous;

- $t_{0}=1$ is not a $(P, S)$-focal instant;

- the equation $V^{\prime \prime}=R V$ admits a solution $Y$ satisfying $g(Y, Y)<0$ on $[0,1]$;

Let $\mathcal{K}$ be the subspace of $H_{P}^{1}\left([0,1], \mathbb{R}^{n}\right)$ consisting of those $V$ 's such that the quantity 
$g\left(V^{\prime}, Y\right)-g\left(V, Y^{\prime}\right)$ is constant a.e. on $[0,1]$. Then

$$
n_{-}\left(I_{1} \mid \mathcal{K}\right)=n_{-}\left(\left.g\right|_{P}\right)+\mathrm{i}_{\mathrm{M}}
$$

where $\mathrm{i}_{\mathrm{M}}=\mathrm{i}_{\mathrm{M}}(g, R, P, S)$ is the Maslov index of the Morse-Sturm problem and $I_{1}$ is the bilinear form on $H_{P}^{1}\left([0,1], \mathbb{R}^{n}\right)$ defined in (3.9).

6. The Lorentzian Morse Index Theorem. The main motivation for studying extensions of the Morse-Sturm theory in the case of non positive metrics $g$ comes from the applications to the geodesic problem in semi-Riemannian geometry. In this section we discuss the case of Lorentzian manifolds, and in particular we show how Theorem 5.1 can be interpreted as a generalization of the classical Morse Index Theorem.

We introduce the following geometrical setup.

Let's assume that $(\mathcal{M}, g)$ is a Lorentzian manifold, $n=\operatorname{dim}(\mathcal{M})$, and that $\gamma$ : $[0,1] \mapsto \mathcal{M}$ is a geodesic, i.e., $\nabla_{\dot{\gamma}} \dot{\gamma}=0$, where $\nabla$ is the covariant derivative of the Levi-Civita connection of $g$. We denote by $R$ the curvature tensor of $\nabla$, chosen with the following sign convention: $R(X, Y)=\nabla_{X} \nabla_{Y}-\nabla_{Y} \nabla_{X}-\nabla_{[X, Y]}$.

Let $\mathcal{P}$ be a smooth submanifold of $\mathcal{M}$, with $\gamma(0) \in \mathcal{P}, \dot{\gamma}(0) \in T_{\gamma(0)} \mathcal{P}^{\perp}, \gamma(1)=q$, and assume that $g$ is non degenerate on $T_{\gamma(0)} \mathcal{P}$; we say that $\mathcal{P}$ is non degenerate at $\gamma(0)$. The second fundamental form of $\mathcal{P}$ at $\gamma(0)$ in the direction $n$ is the symmetric bilinear form $S_{n}: T_{\gamma(0)} \mathcal{P} \times T_{\gamma(0)} \mathcal{P} \mapsto \mathbb{R}$ given by:

$$
S_{n}\left(v_{1}, v_{2}\right)=g\left(\nabla_{v_{1}} V_{2}, n\right)
$$

where $V_{2}$ is any extension of $v_{2}$ to a vector field on $\mathcal{P}$. Since $g$ is non degenerate on $T_{\gamma(0)} \mathcal{P}$, then there exists a linear operator, still denoted by $S_{n}$, on $T_{\gamma(0)} \mathcal{P}$, such that $S_{n}\left(v_{1}, v_{2}\right)=g\left(S_{n}\left[v_{1}\right], v_{2}\right)$ for all $v_{1}, v_{2} \in T_{\gamma(0)} \mathcal{P}$.

A Jacobi field along $\gamma$ is a smooth vector field $J$ along $\gamma$ that satisfies the Jacobi equation

$$
\nabla_{\dot{\gamma}}^{2} J+R(\dot{\gamma}, J) \dot{\gamma}=0
$$

a $\mathcal{P}$-Jacobi field is a Jacobi field $J$ along $\gamma$ that satisfies the initial conditions:

$$
J(0) \in T_{\gamma(0)} \mathcal{P}, \quad\left[\nabla_{\dot{\gamma}(0)} J+S_{\dot{\gamma}(0)}[J(0)]\right] \in T_{\gamma(0)} \mathcal{P}^{\perp} .
$$

The index form $I_{\{\gamma, \mathcal{P}\}}$ is the symmetric bilinear form defined on the vector space $\mathcal{H}_{\{\gamma, \mathcal{P}\}}$ consisting of those piecewise smooth vector fields $V$ along $\gamma$ such that $V(0) \in$ $T_{\gamma(0)} \mathcal{P}$ and $V(1)=0$, defined by:

$$
\begin{aligned}
I_{\{\gamma, \mathcal{P}\}}(V, W)= & \int_{0}^{1}\left[g\left(\nabla_{\dot{\gamma}} V, \nabla_{\dot{\gamma}} W\right)+g(R(\dot{\gamma}, V) \dot{\gamma}, W)\right] \mathrm{d} t \\
& -g\left(S_{\dot{\gamma}(0)}[V(0)], W(0)\right) .
\end{aligned}
$$

It is easy to see that a vector field $V \in \mathcal{H}_{\{\gamma, \mathcal{P}\}}$ is a $\mathcal{P}$-Jacobi field if and only if it is in the kernel of $I_{\{\gamma, \mathcal{P}\}}$. A point $\gamma\left(t_{0}\right)$ is said to be a $\mathcal{P}$-focal point along $\gamma$ if there exists a non zero $\mathcal{P}$-Jacobi field along $\gamma$ vanishing at $t_{0}$; the multiplicity of a $\mathcal{P}$-focal point is the dimension of the vector space of all $\mathcal{P}$-Jacobi fields along $\gamma$ vanishing at $t_{0}$. If the initial submanifold $\mathcal{P}$ reduces to a fixed point of $\mathcal{M}$, in which case the $\mathcal{P}$-Jacobi fields along $\gamma$ are simply the Jacobi fields vanishing at $t=0$, then the focal points 
are also called conjugate points. If $\gamma$ is either timelike or lightlike, in which case $\mathcal{P}$ is necessarily a spacelike submanifold of $\mathcal{M}$ at $\gamma(0)$, then there are only a finite number of $\mathcal{P}$-focal points along $\gamma$, and their number, with multiplicity, is defined to be the geometric index of the geodesic $\gamma$ (see [19]).

The geodesic $\gamma$ is a critical point of the action functional:

$$
f(z)=\frac{1}{2} \int_{0}^{1} g(\dot{z}, \dot{z}) \mathrm{d} t,
$$

defined on the set $\Omega_{\{\mathcal{P}, q\}}$ of paths $z:[0,1] \mapsto \mathcal{M}$ such that $z(0) \in \mathcal{P}$ and $z(1)=q$; the space $\mathcal{H}_{\{\gamma, \mathcal{P}\}}$ can be seen as the tangent space of $\Omega_{\{\mathcal{P}, q\}}$ at $\gamma$ and the bilinear form $I_{\{\gamma, \mathcal{P}\}}$ is the second variation of $f$ at $\gamma$. Hence, the index of $I_{\{\gamma, \mathcal{P}\}}$ in $\mathcal{H}_{\{\gamma, \mathcal{P}\}}$ is the Morse index of the functional $f$ at the critical point $\gamma$; moreover, $\gamma$ is a non degenerate critical point of $f$ precisely when the point $q$ is not $\mathcal{P}$-focal along $\gamma$.

The Morse index of $f$ at $\gamma$ is not finite, due to the indefiniteness of the metric $g$. However, the theory developed in the previous sections indicate that we can determine a finite index carrying some geometric information about $\gamma$ provided that we restrict the bilinear form $I_{\{\gamma, \mathcal{P}\}}$ to a suitable subspace of $\mathcal{H}_{\{\gamma, \mathcal{P}\}}$.

To describe how the geometrical problem fits into the theory of Morse-Sturm systems discussed in the previous sections, we consider a trivialization of the tangent bundle $T \mathcal{M}$ along $\gamma$ by means of a family $\left\{E_{1}, \ldots, E_{n}\right\}$ of parallel vector fields along $\gamma$.

The map $V=\sum_{i} \lambda_{i} \cdot E_{i} \mapsto\left(\lambda_{1}, \ldots, \lambda_{n}\right)$ gives an isomorphism of $\mathcal{H}_{\{\gamma, \mathcal{P}\}}$ with the vector space of all piecewise smooth $\mathbb{R}^{n}$-valued functions on $[0,1]$. Since each $E_{i}$ is parallel, the covariant derivative of vector fields along $\gamma$ correspond to the usual differentiation in $\mathbb{R}^{n}$; moreover, the Lorentzian metric $g$ is carried to a constant nondegenerate bilinear form on $\mathbb{R}^{n}$, still denoted by $g$, with $n_{-}(g)=1$. For each $t \in[0,1]$, the map

$$
\mathbb{R}^{n} \simeq T_{\gamma(t)} \mathcal{M} \ni v \mapsto R(\dot{\gamma}(t), v) \dot{\gamma}(t) \in T_{\gamma(t)} \mathcal{M} \simeq \mathbb{R}^{n}
$$

is given by a $g$-symmetric linear operator on $\mathbb{R}^{n}$, still denoted by $R(t)$. Finally, the tangent space $T_{\gamma(0)} \mathcal{P}$ corresponds to a $g$-nondegenerate subspace $P$ of $\mathbb{R}^{n}$, and the second fundamental form $S_{\dot{\gamma}(0)}$ gives a $g$-symmetric linear map $S: P \mapsto P$.

The bilinear form $I_{\{\gamma, \mathcal{P}\}}$ is carried into the bilinear form $I_{1}$, defined in the set of piecewise smooth $\mathbb{R}^{n}$-valued functions on [0,1], given by formula (3.9). Since $I_{1}$ has a continuous extension to the Hilbert space $H_{P}^{1}\left([0,1], \mathbb{R}^{n}\right)$, an easy density argument shows that the index of $I_{\{\gamma, \mathcal{P}\}}$ on $\mathcal{H}_{\{\gamma, \mathcal{P}\}}$ is equal to the index of $I_{1}$ on $H_{P}^{1}\left([0,1], \mathbb{R}^{n}\right)$. The Jacobi equation (6.1) becomes the Morse-Sturm system (3.1), the initial conditions (6.2) are read into (3.2), and we have translated our Lorentzian geodesic problem into the Morse-Sturm problem (3.1) and (3.2).

Clearly, the space $\mathbb{J}$ defined in (3.3) corresponds to the set $\mathcal{J}_{\mathcal{P}}$ of $\mathcal{P}$-Jacobi fields, and the $(P, S)$-focal instants are precisely the $\mathcal{P}$-focal points along $\gamma$. The space $\mathcal{J}_{\mathcal{P}}[t] \subset T_{\gamma(t)} \mathcal{M}$ is defined to be the set of values at $t$ of the fields in $\mathcal{J}_{\mathcal{P}}$; the signature $\operatorname{sgn}\left(\gamma\left(t_{0}\right)\right)$ of the $\mathcal{P}$-focal point $\gamma\left(t_{0}\right)$ is defined to be the signature of the metric $g$ on the space $\mathcal{J}_{\mathcal{P}}\left[t_{0}\right]^{\perp}$; the $\mathcal{P}$-focal point $\gamma\left(t_{0}\right)$ is said to be positive, null or negative according to whether $\operatorname{sgn}\left(\gamma\left(t_{0}\right)\right)$ is positive, null or negative. ${ }^{3}$

\footnotetext{
${ }^{3}$ The reader should observe that we are using a terminology slightly different from the one adopted in [12], where it is defined a timelike, a null and a spacelike index for each conjugate point.
} 
The important observation here is that, if $\gamma$ is causal, i.e., timelike or lightlike, then the restriction of the metric $g$ to the space $\mathcal{J}_{\mathcal{P}}[t]^{\perp}$ is always positive definite, so that the signature of a $\mathcal{P}$-focal point coincides with its multiplicity. This implies in particular that the Maslov index of $\gamma$ coincides precisely with the geometrical index of $\gamma$.

Under the assumption that the point $\gamma(1)$ is not $\mathcal{P}$-focal along $\gamma$, we can therefore apply Theorem 5.1 to the geometrical problem, obtaining the following generalization of the Morse Index Theorem for Lorentzian geodesics with variable initial endpoint:

THEOREM 6.1. Let $(\mathcal{M}, g)$ be a Lorentzian manifold, $\mathcal{P} \subset \mathcal{M}$ a smooth submanifold, $\gamma:[0,1] \mapsto \mathcal{M}$ a geodesic with $\gamma(0) \in \mathcal{P}$ and $\dot{\gamma}(0) \in T_{\gamma(0)} \mathcal{P}^{\perp}$. Assume that the following hypotheses are satisfied:

- there exists a timelike Jacobi field $Y$ along $\gamma$;

- $\mathcal{P}$ is non degenerate at $\gamma(0)$;

- $\gamma(1)$ is not $\mathcal{P}$-focal along $\gamma$.

Then, denoting by $\mathcal{K}^{\gamma}$ the space of (piecewise smooth) vector fields $V$ along $\gamma$ satisfying $V(0) \in T_{\gamma(0)} \mathcal{P}, V(1)=0$ and $g\left(\nabla_{\dot{\gamma}} V, Y\right)-g\left(V, \nabla_{\dot{\gamma}} Y\right) \equiv C_{V}$ (constant), the index of $I_{\{\gamma, \mathcal{P}\}}$ on $\mathcal{K}^{\gamma}$ is finite, and the following equality holds:

$$
n_{-}\left(\left.I_{\{\gamma, \mathcal{P}\}}\right|_{\mathcal{K} \gamma}\right)=n_{-}\left(\left.g\right|_{T_{\gamma(0)} \mathcal{P}}\right)+\mathrm{i}_{\mathrm{M}}(\gamma) .
$$

Moreover, if $\gamma$ is causal, then $\mathrm{i}_{\mathrm{M}}(\gamma)$ equals the geometric index of $\gamma . \square$

Observe that the quantity on the right hand side of (6.5) does not depend on the choice of the timelike Jacobi field $Y$, hence the index of $I_{\{\gamma, \mathcal{P}\}}$ on the space $\mathcal{K}^{\gamma}$ is also independent on the choice of $Y$. We also remark that, if $\gamma$ is a timelike geodesic, then one can take as a timelike Jacobi field $Y$ the tangent field $\dot{\gamma}$. It is easy to see that, in this case, the space $\mathcal{K}^{\gamma}$ consists precisely of those vector fields along $\gamma$ that are pointwise orthogonal to $\dot{\gamma}$. Hence, Theorem 6.1 gives a generalization of the Timelike Morse Index Theorem of [3, Theorem 10.27].

An important class of examples where the assumption on the existence of a timelike Jacobi field along any geodesic is satisfied is given by the stationary Lorentzian manifolds, i.e., Lorentzian manifolds admitting a timelike Killing vector field. In this case, a timelike Jacobi vector field along every geodesic is given by the restriction of any timelike Killing field (see [18, Lemma 9.26, p. 252]).

It is interesting to observe that, for non positive definite metrics, as we can deduce from equation (6.5), the Morse index of the action functional at a given geodesic $\gamma$ may be strictly positive even in the case that $\gamma$ has no focal points. This happens precisely when the initial submanifold $\mathcal{P}$ is non spacelike. For a better understanding of this fact, one can consider the following simple but instructive example.

ExAmPle 6.2. Let $(\mathcal{M}, g)$ be the two-dimensional flat Minkowski space, with metric $\mathrm{d} x^{2}-\mathrm{d} y^{2}$. Let $\gamma(t)=(t, 0), t \in[0,1]$, and let $\mathcal{P}$ denote the one-dimensional timelike submanifold of $\mathcal{M}$ given by the $y$-axis; we are in the situation described in the hypotheses of Theorem 6.1, considering $Y=\frac{\partial}{\partial y}$ as the timelike Jacobi field along $\gamma$. Clearly, there are no $\mathcal{P}$-focal points along $\gamma$, and both the curvature tensor $R$ of $g$ and the second fundamental form $S$ of $\mathcal{P}$ are null.

We have $n_{-}\left(\left.g\right|_{T_{(0,0)} \mathcal{P}}\right)=1$; the space $\mathcal{K}^{\gamma}$ consists of vector fields of the form $V=a(t) \frac{\partial}{\partial x}+b(t) \frac{\partial}{\partial y}$, with $a(0)=a(1)=b(1)=0$ and $b^{\prime}(t) \equiv C_{V}$ constant on $[0,1]$. 
For $V \in \mathcal{K}^{\gamma}$, the value of the index form $I_{\{\gamma, \mathcal{P}\}}(V, V)$ is computed easily as:

$$
I_{\{\gamma, \mathcal{P}\}}(V, V)=\int_{0}^{1}\left[a^{\prime}(t)^{2}-b^{\prime}(t)^{2}\right] \mathrm{d} t=\int_{0}^{1} a^{\prime}(t)^{2} \mathrm{~d} t-C_{V}^{2} .
$$

If we consider $a \equiv 0$, we get a one-dimensional subspace of $\mathcal{K}^{\gamma}$ on which $I_{\{\gamma, \mathcal{P}\}}$ is negative definite; on the other hand, if we consider $b \equiv 0$ and $a$ arbitrary, we get a complementary subspace where $I_{\{\gamma, \mathcal{P}\}}$ is positive definite, thus $n_{-}\left(\left.I_{\{\gamma, \mathcal{P}\}}\right|_{\mathcal{K}^{\gamma}}\right)=1$.

It is fairly easy to give examples of $\mathcal{P}$-focal points in stationary Lorentzian manifolds of every causal type. Examples of positive focal or conjugate points are easily constructed by considering causal geodesics, or spacelike geodesics admitting a parallel timelike Jacobi field along them (see Example 6.5 below). In the next example we construct elementary examples of negative and null focal points in manifolds with flat metric.

ExAmple 6.3. Consider the Minkowski plane $\mathbb{R}^{2}$ endowed with the flat metric $g=\mathrm{d} x^{2}-\mathrm{d} y^{2}$; let $\gamma(t)=(t, 0)$ be the (spacelike) geodesic segment on the $x$-axis, and let $\mathcal{P}$ denote the parabola through the origin given by the equation $y^{2}+2 x=0$. Then, $\gamma$ is orthogonal to $\mathcal{P}$ at $(0,0)=\gamma(0)$; the second fundamental form of $\mathcal{P}$ at $(0,0)$ is easily computed as

$$
S_{\dot{\gamma}(0)}\left(\frac{\partial}{\partial y}\right)=\frac{\partial}{\partial y}
$$

so that $J(t)=(t-1) \frac{\partial}{\partial y}$ is a $\mathcal{P}$-Jacobi field along $\gamma$ which vanishes at $t=1$. Clearly, $\gamma(1)=(1,0)$ is a $\mathcal{P}$-focal point of multiplicity one along $\gamma$, and $\operatorname{sgn}(\gamma(1))=$ $\operatorname{sgn}\left(\left.g\right|_{\mathbb{R} \cdot J^{\prime}(1)}\right)=\operatorname{sgn}\left(\left.g\right|_{\mathbb{R} \cdot \frac{\partial}{\partial y}}\right)=-1$.

To construct an example of a null $\mathcal{P}$-focal point we now consider the threedimensional flat Minkowski space $\mathcal{M}=\mathbb{R}^{3}$ with metric $g=\mathrm{d} x^{2}+\mathrm{d} y^{2}-\mathrm{d} z^{2}$ and the spacelike geodesic $\gamma(t)=(t, 0,0), t \in[0,1]$. Let $\mathcal{P}$ be any smooth surface through the origin such that the tangent plane $T_{(0,0,0)} \mathcal{P}$ is the $y z$-plane and such that the second fundamental form $S_{\dot{\gamma}(0)}$ of $\mathcal{P}$ at $(0,0,0)$ satisfies $^{4}$

$$
S_{\dot{\gamma}(0)}\left(\frac{\partial}{\partial y}+\frac{\partial}{\partial z}\right)=\frac{\partial}{\partial y}+\frac{\partial}{\partial z} .
$$

Arguing as before it is easy to verify that $J(t)=(t-1)\left(\frac{\partial}{\partial y}+\frac{\partial}{\partial z}\right)$ is a $\mathcal{P}$-Jacobi field along $\gamma, J(1)=0, J^{\prime}(1)$ is the lightlike vector $\frac{\partial}{\partial y}+\frac{\partial}{\partial z}$, and $\gamma(1)$ is a null $\mathcal{P}$-focal point along $\gamma$.

REMARK 6.4. Theorem 6.1 can be extended to the case of geodesics in semi-Riemannian manifolds $(\mathcal{M}, g)$, with $g$ of arbitrary index $n_{-}(g)=k \geq 1$. In this case, given a geodesic $\gamma$ in $\mathcal{M}$, one needs to assume the existence of $k$ Jacobi fields $J_{1}, \ldots, J_{k}$ along $\gamma$ generating a $k$-dimensional timelike distribution along $\gamma$, and satisfying the relations $g\left(\nabla_{\dot{\gamma}} Y_{i}, Y_{j}\right)-g\left(Y_{i}, \nabla_{\dot{\gamma}} Y_{j}\right)=0$ for all $i, j=1, \ldots, k$. One considers the space $\mathcal{K}^{\gamma}$ of vector fields $V$ along $\gamma$ satisfying $V(0) \in T_{\gamma(0)} \mathcal{P}, V(1)=0$ and $g\left(\nabla_{\dot{\gamma}} V, Y_{i}\right)-$

\footnotetext{
${ }^{4}$ of course, such submanifold $\mathcal{P}$ exists; see for instance [16, Lemma 2.3.2] for details on how to construct a smooth submanifold of a semi-Riemannian manifold when its tangent space and its second fundamental form is assigned at one point.
} 
$g\left(V, \nabla_{\dot{\gamma}} Y_{i}\right) \equiv C_{V}^{(i)}$ (const.) for all $i=1, \ldots, k$. Then, if $\gamma(1)$ is not $\mathcal{P}$-focal, the index of $I_{\{\gamma, \mathcal{P}\}}$ on $\mathcal{K}^{\gamma}$ equals $\mathrm{i}_{\mathrm{M}}(\gamma)+n_{-}\left(\left.g\right|_{T_{\gamma(0)} \mathcal{P}}\right)$.

Examples of semi-Riemannian manifolds where the theory applies are given by those manifolds admitting a family of Killing vector fields $Y_{1}, \ldots, Y_{k}$ that generate a $k$-dimensional timelike distribution on $\mathcal{M}$, and satisfying the commutation relations $\left[Y_{i}, Y_{j}\right]=0$ for all $i, j=1, \ldots, k$. A variational theory for geodesics in this kind of manifolds is presented in [11]. All the results presented in this paper can be extended to this more general situation.

We now discuss the case of conjugate points along Lorentzian geodesics satisfying the hypotheses of Theorem 6.1 , and so we assume that the initial manifold $\mathcal{P}$ reduces to a single point. This means that the $\mathcal{P}$-Jacobi fields along $\gamma$ are simply the Jacobi fields vanishing at $t=0$. We denote by $I_{\gamma}$ the index form along $\gamma$ relative to the choice of a trivial initial manifold.

The first observation is that, in this situation, if $Y$ is parallel along the geodesic $\gamma$, then the conjugate points along $\gamma$ are isolated, and they are all positive.

ExAmple 6.5. Suppose that $Y$ is a parallel timelike Jacobi field along the geodesic $\gamma$; this means that $\nabla_{\dot{\gamma}} Y=0$, and so $\nabla_{\dot{\gamma}}^{2} Y=R(\dot{\gamma}, Y) \dot{\gamma}=0$.

If $J$ is Jacobi, then $g\left(\nabla_{\dot{\gamma}} J, Y\right)-g\left(J, \nabla_{\dot{\gamma}} Y\right)=g\left(\nabla_{\dot{\gamma}} J, Y\right)$ is constant on [0,1], hence $\frac{\mathrm{d}^{2}}{\mathrm{~d} t^{2}} g(J, Y)=g\left(\nabla_{\dot{\gamma}}^{2} J, Y\right)=0$, and $g(J, Y)$ is an affine function on [0,1].

If $\gamma\left(t_{0}\right)$ is conjugate to $\gamma(0)$ along $\gamma$, and $J$ is a non trivial Jacobi field along $\gamma$ vanishing at 0 and $t_{0}$, then it must be $g(J, Y) \equiv 0$, and so $g\left(\nabla_{\dot{\gamma}} J, Y\right) \equiv 0$. It is $\mathcal{J}_{\mathcal{P}}\left[t_{0}\right]^{\perp}=\left\{\nabla_{\dot{\gamma}} J\left(t_{0}\right): J\right.$ Jacobi, with $\left.J(0)=J\left(t_{0}\right)=0\right\}$, and it follows that $\mathcal{J}_{\mathcal{P}}\left[t_{0}\right]^{\perp} \subset Y\left(\gamma\left(t_{0}\right)\right)^{\perp}$. Since $Y$ is timelike, it follows that the restriction of the metric $g$ to $\mathcal{J}_{\mathcal{P}}\left[t_{0}\right]^{\perp}$ is positive definite, which implies that the conjugate point $\gamma\left(t_{0}\right)$ is isolated and that its signature $\operatorname{sgn}\left(\gamma\left(t_{0}\right)\right)$ is equal to its multiplicity. Hence, the Maslov index of $\gamma$ coincides with its geometric index. In this case, Theorem 6.1 tells us that, if $\gamma(1)$ is not conjugate to $\gamma(0)$ along $\gamma$, the index of $I_{\gamma}$ on $\mathcal{K}^{\gamma}$ is equal to the geometric index of $\gamma$.

Let's assume now that the geodesic $\gamma$ satisfies the assumptions of Theorem 6.1 and the non degeneracy assumption of Proposition 3.1. It is an easy observation that there cannot be too many negative conjugate points along $\gamma$. For example, if $\gamma\left(t_{0}\right)$ is a negative conjugate point, i.e., $\operatorname{sgn}\left(\gamma\left(t_{0}\right)\right)=-1$, then the Maslov index $\mathrm{i}_{\mathrm{M}}\left(\left.\gamma\right|_{\left[0, t_{0}-\varepsilon\right]}\right)$ must be strictly positive for $\varepsilon>0$ small enough. This follows immediately from the fact that, by Theorem 6.1 , if $\varepsilon>0$ is small enough, it must be

$$
n_{-}\left(\left.I_{\gamma}\right|_{\mathcal{K}_{t_{0}+\varepsilon}^{\gamma}}\right)=\mathrm{i}_{\mathrm{M}}\left(\left.\gamma\right|_{\left[0, t_{0}+\varepsilon\right]}\right)=\mathrm{i}_{\mathrm{M}}\left(\left.\gamma\right|_{\left[0, t_{0}-\varepsilon\right]}\right)-1 \geq 0 .
$$

In particular, the first conjugate point along $\gamma$ is never negative.

If $\operatorname{dim}(\mathcal{M})=2$, then the metric $-g$ is Lorentzian in $\mathcal{M}$. This simple observation allows to get some interesting consequences, like the following:

Proposition 6.6. Let $(\mathcal{M}, g)$ be a two dimensional Lorentzian manifold and let $\gamma:[0,1] \mapsto \mathcal{M}$ be a spacelike geodesic in $\mathcal{M}$. Suppose that there exists a timelike Jacobi field along $\gamma$. Then, there are no conjugate points along $\gamma$.

Proof. The curve $\gamma$ is clearly a timelike geodesic in the opposite Lorentzian manifold $(\mathcal{M},-g)$ with the same conjugate points. We know that all the conjugate points along a causal geodesic are positive, hence $\gamma$ has only negative conjugate points in $(\mathcal{M}, g)$. Then, there cannot be any conjugate point, because the sum of their signatures must be non negative integer. 
By the same argument, it is easy to see that if $\gamma$ is a spacelike geodesic in a two-dimensional Lorentzian manifold $\mathcal{M}$, starting orthogonally to a one-dimensional (necessarily timelike) submanifold $\mathcal{P}$ of $\mathcal{M}$, then there is at the most one $\mathcal{P}$-focal point along $\gamma$, which must be negative (see Example 6.3). It is well known that conjugate points cannot occur along lightlike geodesics in two-dimensional Lorentzian manifolds (see [3]). However, we remark that spacelike (or timelike) geodesics in two-dimensional Lorentzian Lorentzian manifolds may have conjugate points. For instance, in the conformally flat metric $e^{t^{2}}\left(\mathrm{~d} x^{2}-\mathrm{d} t^{2}\right)$ on $\mathbb{R}^{2}$, the curve $\gamma(\tau)=(\tau, 0)$ is a spacelike geodesic, and the Jacobi equation along $\gamma$ is for the vector field $J=(v, w)$ is given by the system

$$
v^{\prime \prime}=0, \quad w^{\prime \prime}+w=0 .
$$

Clearly, the point $\gamma(\pi)$ is conjugate to $\gamma(0)$ along $\gamma$.

We leave unanswered the following questions:

1. do there exist examples of (spacelike) Lorentzian geodesics satisfying the hypotheses of Theorem 6.1 for which the set of $\mathcal{P}$-focal (or conjugate) points is not discrete?

2. can a (spacelike) Lorentzian geodesic satisfying the hypotheses of Theorem 6.1 really have one negative conjugate point?

3. suppose that $\gamma$ is a (spacelike) geodesic satisfying the hypotheses of Theorem 6.1 and having one or more isolated conjugate point for which the non degeneracy assumption of Proposition 3.1 is not satisfied; is it still true that the Maslov index of $\gamma$ is given by the sum of the signatures of its conjugate points?

If one does not require the assumptions of Theorem 6.1 all the above questions have easy answers (see [16]): the first two questions have a positive answer and the third one has a negative answer.

We conclude this section with the remark that a Lorentzian version of the Morse Index Theorem for the two variable endpoints (see [13] for the Riemannian case) can be easily deduced from Theorem 6.1. When the final endpoint of $\gamma$ is allowed to vary on a submanifold $\mathcal{Q}$ of $\mathcal{M}$, the index of the second variation of the action functional at $\gamma$ is given by the sum of the right hand side of equation (6.5) and a term that measures the relative convexity of $\mathcal{Q}$ with respect to $\mathcal{P}$. The details are found in [19, Theorem 2.7, Remark 2.10].

7. The Global Morse Relations for Geodesics in Stationary Lorentzian Manifolds. In this section we want to develop an infinite dimensional Morse theory for the geodesics joining two fixed points $p$ and $q$ in a stationary Lorentzian manifold $(\mathcal{M}, g)$, in the spirit of [17] and using the modern terminology of [5]. The main goal of this theory is to give estimates on the number of geodesics having a given index; these estimates are given in terms of the topology of the space of (continuous) curves joining $p$ and $q$ in $\mathcal{M}$. The basic reference for most of the material discussed in this section is [10]; we will make full use of the results proven in that article.

As customary, if $I \subseteq \mathbb{R}$ is any interval, we will denote by $H^{1}\left(I, \mathbb{R}^{n}\right)$ the Sobolev space of absolutely continuous curves $z: I \longmapsto \mathbb{R}^{n}$ such that the integral $\int_{I}|\dot{z}|^{2} \mathrm{~d} t$ is finite, where $|\cdot|$ denotes the Euclidean norm in $\mathbb{R}^{n}$.

Given any differentiable manifold $N$, the set $H^{1}([0,1], N)$ is defined as the set of all absolutely continuous curves $z:[0,1] \longmapsto N$ such that, for every local chart $(V, \varphi)$ on $N$, with $\varphi: U \longmapsto \mathbb{R}^{n}$ a diffeomorphism, and for every closed subinterval 
$I \subseteq[0,1]$ such that $z(I) \subset V$, it is $\varphi \circ z \in H^{1}\left(I, \mathbb{R}^{n}\right)$. For all differentiable manifold $N$, with $\operatorname{dim}(N)=n$, the set $H^{1}([0,1], N)$ has the structure of an infinite dimensional manifold, modeled on the Hilbert space $H^{1}\left([0,1], \mathbb{R}^{n}\right)$. We will denote by $T N$ the tangent bundle of $N$ and by $\pi: T N \mapsto N$ the canonical projection; for $p \in N$, $T_{p} N=\pi^{-1}(p)$ denotes the tangent space of $N$ at $p$. A vector field along a curve $z:[0,1] \mapsto N$ is a map $\zeta:[0,1] \mapsto T N$ with $\pi(\zeta(t))=z(t)$ for all $t$. Given any $z \in H^{1}([0,1], N)$, the tangent space $T_{z} H^{1}([0,1], N)$ is identified with the set:

$$
T_{z} H^{1}([0,1], N)=\left\{\zeta \in H^{1}([0,1], T N): \zeta \text { vector field along } z\right\}
$$

which is an infinite dimensional vector space, with a topology that makes it into a Hilbertable space.

Let's assume that $(\mathcal{M}, g)$ is a Lorentzian manifold which admits a timelike Killing vector field, denoted by $Y$. We assume that $Y$ is complete; let $p$ and $q$ be fixed points in $\mathcal{M}$. We introduce the following space:

$$
\Omega_{p, q}=\left\{z \in H^{1}([0,1], \mathcal{M}): z(0)=p, z(1)=q\right\}
$$

It is well known that $\Omega_{p, q}$ has the structure of an infinite dimensional Hilbertian submanifold of $H^{1}([0,1], \mathcal{M})$; the action functional $f$, defined in (6.4), is smooth on $\Omega_{p, q}$ and its critical points are precisely the geodesics in $\mathcal{M}$ between $p$ and $q$. We say that $p$ and $q$ are non conjugate in $\mathcal{M}$ if they are not conjugate along every geodesic in $\mathcal{M}$ joining them.

For all geodesic $\gamma$ in $\mathcal{M}$ we have a conservation law $g(\dot{\gamma}, Y) \equiv c_{\gamma}$ (constant). Now, if we consider the subset $\Omega_{p, q}^{Y}$ of $\Omega_{p, q}$ consisting of curves $z$ satisfying $g(\dot{z}, Y) \equiv$ const., then clearly the geodesics in $\Omega_{p, q}$ belong to $\Omega_{p, q}^{Y}$. It is proven in [10] that $\Omega_{p, q}^{Y}$ is a smooth submanifold of $\Omega_{p, q}$, and that $f$ has the same critical points in $\Omega_{p, q}$ and in $\Omega_{p, q}^{Y}$.

By differentiating the expression $g(\dot{z}, Y)=$ const. with respect to $z$, using the Killing property of $Y$ it is easy to see that the tangent space $T_{z} \Omega_{p, q}^{Y}$ is given by the Hilbert space of $H^{1}$-vector fields along $z$ satisfying $V(0)=V(1) \stackrel{p, q}{=} 0$ and such that the quantity $g\left(\nabla_{\dot{z}} V, Y\right)-g\left(V, \nabla_{\dot{z}} Y\right)$ is constant a.e. on $[0,1]$.

Hence, if $\gamma$ is a critical point for $f$ in $\Omega_{p, q}^{Y}$, i.e., a geodesic between $p$ and $q$, the tangent space $T_{\gamma} \Omega_{p, q}^{Y}$ is a completion of the space $\mathcal{K}^{\gamma}$ of Theorem 6.1 , and the index of $I_{\gamma}$ in $\mathcal{K}^{\gamma}$ is equal to the Morse index of the functional $f$ at the critical point $\gamma \in \Omega_{p, q}^{Y}$.

Such index can therefore be interpreted as the number of essentially different directions in which $\gamma$ can be deformed, in the class of curves $z$ joining $p$ with $q$ and satisfying $g(\dot{z}, Y)=$ const., in order to obtain a curve with smaller action.

Let $\mathcal{C}_{p, q}^{1}$ denote the following space:

$$
\begin{aligned}
\mathcal{C}_{p, q}^{1}=\{z:[0,1] & \mapsto \mathcal{M} \text { piecewise } C^{1}: \\
& \left.z(0)=p, z(1)=q, g(\dot{z}, Y) \equiv c_{z} \text { (constant) }\right\} ;
\end{aligned}
$$

we give the following completeness condition for the sublevels of the restricted action functional.

Definition 7.1. Given $c \in \mathbb{R}$, we say that $\mathcal{C}_{p, q}^{1}$ is c-precompact if every sequence $\left\{z_{n}\right\}_{n \in \mathbb{N}} \subset \mathcal{C}_{p, q}^{1}$ such that $f\left(z_{n}\right) \leq c$ has a uniformly convergent subsequence.

The $c$-precompactness property, which is given intrinsically in Definition 7.1, can be studied by means of suitable bounds of the metric coefficients with respect to the 
coordinates of a given atlas on $\mathcal{M}$. A wide class of examples of stationary Lorentzian manifolds $(\mathcal{M}, g)$ for which the $c$-precompactness assumption is satisfied by all choices of $p, q$ and $c$ is given in [10]. We emphasize that the $c$-precompactness for stationary Lorentzian manifold plays the role of the completeness assumption in Riemannian geometry; for this and other analogies with the classical Riemannian theory we refer to [10], where it is also discussed the relation between the $c$-precompactness and the property of global hyperbolicity.

We recall that, given a topological space $X$, an algebraic field $\mathbb{K}$ and a natural number $i$, the $i$-th Betti number $\beta_{i}(X ; \mathbb{K})$ of $X$ relative to $\mathbb{K}$ is the $\mathbb{K}$-dimension of the $i$-th singular homology vector space $H_{i}(X ; \mathbb{K})$ of $X$ with coefficients in $\mathbb{K}$. The Poincaré polynomial $\mathfrak{P}_{\lambda}(X ; \mathbb{K})$ of $X$ with coefficients in $\mathbb{K}$ is the formal power series in $\lambda \in \mathbb{K}$ given by:

$$
\mathfrak{P}_{\lambda}(X ; \mathbb{K})=\sum_{i=0}^{\infty} \beta_{i}(X ; \mathbb{K}) \lambda^{i}
$$

The global Morse relations provide relations between the set of all the geodesics joining $p$ and $q$ in $\mathcal{M}$ with the topology of the space of all continuous curves joining $p$ and $q$ in $\mathcal{M}$, given in terms of the Betti numbers and the Poincaré polynomial of this space. A key point for the infinite dimensional Morse theory is the so called Palais-Smale condition. We recall that a smooth functional $F$ on a manifold $X$ endowed with a Finsler structure is said to satisfy the Palais-Smale condition at the level $c \in \mathbb{R}$ if every sequence $\left\{x_{n}\right\}_{n \in \mathbb{N}} \subset X$ such that:

(a) $\lim _{n \rightarrow \infty} F\left(x_{n}\right)=c$;

(b) $\lim _{n \rightarrow \infty}\left\|\mathrm{d} F\left(x_{n}\right)\right\|=0$,

has a converging subsequence in $X$.

The $c$-precompactness condition given in Definition 7.1 is the key assumption for the proof of the global Morse relations, which are given in the following

TheOREM 7.2. Let $(\mathcal{M}, g)$ be a Lorentzian manifold. Suppose that $\mathcal{M}$ admits a complete timelike Killing vector field $Y$, and assume that $p$ and $q$ are two points of $\mathcal{M}$ such that the following hypotheses are satisfied:

- $p$ and $q$ are not conjugate in $\mathcal{M}$;

- $\mathcal{C}_{p, q}^{1}$ is c-precompact for all $c \in \mathbb{R}$.

Let $\Omega_{p, q}^{0}$ denote the space of all continuous curves $z:[0,1] \mapsto \mathcal{M}$ joining $p$ and $q$ in $\mathcal{M}$, endowed with the topology of uniform convergence, and let $\mathcal{G}_{p, q}$ denote the set of all geodesics in $\mathcal{M}$ between $p$ and $q$. Then, for all field $\mathbb{K}$ there exists a formal power series $Q_{\mathbb{K}}(\lambda)$ in the variable $\lambda$, with coefficients in $\mathbb{I N} \bigcup\{+\infty\}$ such that the following identity between formal power series is satisfied:

$$
\sum_{z \in \mathcal{G}_{p, q}} \lambda^{\mathrm{i}_{\mathrm{M}}(z)}=\mathfrak{P}_{\lambda}\left(\Omega_{p, q}^{0} ; \mathbb{K}\right)+(1+\lambda) Q_{\mathbb{K}}(\lambda) .
$$

Proof. Let $f_{Y}$ denote the restriction of the action functional $f$ to the manifold $\Omega_{p, q}^{Y}$; as we have observed, $\Omega_{p, q}^{Y}$ is a smooth submanifold of $\Omega_{p, q}$ and the critical points of $f_{Y}$ on $\Omega_{p, q}^{Y}$ are precisely the geodesics joining $p$ and $q$ in $\mathcal{M}$.

We endow $\Omega_{p, q}^{Y}$ with the following Riemannian structure. We consider an auxiliary Riemannian metric $g^{(\mathrm{r})}$ on $\mathcal{M}$, and for all $z \in \Omega_{p, q}^{Y}$ we define a Hilbert space inner 
product $\langle\cdot, \cdot\rangle$ in $T_{z} \Omega_{p, q}^{Y}$ by:

$$
\langle V, V\rangle=\int_{0}^{1} g^{(\mathrm{r})}\left(\nabla_{\dot{z}} V, \nabla_{\dot{z}} V\right) \mathrm{d} t
$$

Using the $c$-precompactness assumption, as well as the density of $\mathcal{C}_{p, q}^{1}$ in $\Omega_{p, q}^{Y}$, the following facts are proven in [10]:

1. $f_{Y}$ is bounded from below, i.e., there exists $D \in \mathbb{R}$ such that $f(z) \geq D$ for all $z \in \Omega_{p, q}^{Y}$;

2. for all $c \in \mathbb{R}$, the sublevel $f_{Y}^{c}=\left\{z \in \Omega_{p, q}^{Y}: f(z) \leq c\right\}$ is a complete metric subspace of $\Omega_{p, q}^{Y}$;

3. for all $c \in \mathbb{R}, f_{Y}$ satisfies the Palais-Smale condition at the level $c$ when $\Omega_{p, q}^{Y}$ is endowed with the Finsler structure given by (7.3).

Finally, the condition that $p$ and $q$ be non conjugate in $\mathcal{M}$ implies that $f_{Y}$ is a Morse functional, i.e., all its critical points in $\Omega_{p, q}^{Y}$ are non degenerate. Namely, as we have already observed, the second variation of $f_{Y}$ at any geodesic $\gamma$ is given by the restriction of the index form $I_{\gamma}$, and its kernel in $T_{\gamma} \Omega_{p, q}^{Y}$ coincides with the set of Jacobi fields along $\gamma$ vanishing at the endpoints. If $p$ and $q$ are non conjugate in $\mathcal{M}$, then $I_{\gamma}$ has trivial kernel, and $f_{Y}$ is a Morse functional.

Then, by standard results of Global Analysis on Manifolds (see for instance [15]), denoting by $m\left(z, f_{Y}\right)$ the Morse index of the critical point $z$ of $f_{Y}$, we have the following Morse relations. For all field $\mathbb{I} K$ there exists a formal power series $Q_{\mathbb{K}}(\lambda)$ in the variable $\lambda$, with coefficients in $\mathbb{I} \bigcup\{+\infty\}$ such that the following identity between formal power series is satisfied:

$$
\sum_{z \in \mathcal{G}_{p, q}} \lambda^{m\left(z, f_{Y}\right)}=\mathfrak{P}_{\lambda}\left(\Omega_{p, q}^{Y} ; \mathbb{K}\right)+(1+\lambda) Q_{\mathbb{K}}(\lambda) .
$$

By Theorem 6.1, for all $z \in \mathcal{G}_{p, q}$ we have $m\left(z, f_{Y}\right)=\mathrm{i}_{\mathrm{M}}(z)$; moreover, since $Y$ is complete, it is proven in [10] that the spaces $\Omega_{p, q}$ and $\Omega_{p, q}^{Y}$ are homotopically equivalent, which implies that $\mathfrak{P}_{\lambda}\left(\Omega_{p, q}^{Y} ; \mathbb{K}\right)=\mathfrak{P}_{\lambda}\left(\Omega_{p, q} ; \mathbb{K}\right)$ for all field $\mathbb{K}$. Finally, also the spaces $\Omega_{p, q}$ and $\Omega_{p, q}^{0}$ have the same homotopy type (see [17]), and so the Morse relations (7.2) are easily obtained from (7.4).

\section{REFERENCES}

[1] V. I. ARnol'D, Characteristic class entering in quantization conditions, Funct. Anal. Appl., 1 (1967), pp. 1-13.

[2] J. Beem And P. Ehrlich, A Morse index theorem for null geodesics, Duke Math. J., 46 (1979), pp. 561-569.

[3] J. K. BeEm, P. E. Ehrlich, And K. L. EAsley, Global Lorentzian Geometry, Marcel Dekker, Inc., New York and Basel, 1996.

[4] V. Benci And A. Masiello, A Morse index for geodesics os static Lorentzian manifolds, Math. Ann., 293 (1992), pp. 433-442.

[5] R. Botr, Lectures on Morse theory, old and new, Bull. Amer. Math. Soc., 7 (1982), pp. 331358.

[6] H. Brezis, Analyse Fonctionelle, Masson, Paris, 1983.

[7] M. Do CARmo, Riemannian Geometry, Birkhäuser, Boston, 1992.

[8] H. M. Edwards, A generalized Sturm theorem, Ann. of Math., 80 (1964), pp. 22-57.

[9] F. Giannoni and A. Masiello, Morse relations for geodesics on stationary Lorentzian manifolds with boundary, Top. Meth. Nonlinear Anal., 6 (1995), pp. 1-30.

[10] F. Giannoni And P. Piccione, An intrinsic approach to the geodesical connectedness of stationary Lorentzian manifolds, Commun. Anal. Geom., 7:1 (1999), pp. 157-197. 
[11] F. Giannoni, P. Piccione, AND R. SAmpalmieri, On the geodesical connectedness for a class of semi-Riemannian manifolds, Journal of Mathematical Analysis and Applications, 252:1 (2000), pp. 444-476.

[12] A. D. HELFER, Conjugate points on spacelike geodesics or pseudo-self-adjoint Morse-SturmLiouville systems, Pacific J. Math., 164:2 (1994), pp. 321-340.

[13] D. KALISH, The Morse index theorem where the ends are submanifolds, Trans. Am. Math. Soc., 308:1 (1988), pp. 341-348.

[14] A. Masiello, Variational Methods in Lorentzian Geometry, Pitman Research Notes in Mathematics 309, Longman, London, 1994.

[15] J. Mawhin and M. Willem, Critical Point Theory and Hamiltonian Systems, Springer-Verlag, Berlin, 1989.

[16] F. Mercuri, P. Piccione, and D. Tausk, Stability of the focal and the geometric index in semi-Riemannian geometry via the Maslov index, Technical Report RT-MAT 99-08, Mathematics Department, University of São Paulo, Brazil, 1999 (LANL math.FA/9905096).

[17] J. Milnor, Morse Theory, Princeton Univ. Press, Princeton, 1969.

[18] B. O'NeILl, Semi-Riemannian Geometry with Applications to Relativity, Academic Press, New York, 1983.

[19] P. Piccione And D. V. TAusk, A note on the Morse index theorem for geodesics between submanifolds in semi-Riemannian geometry, J. Math. Phys., 40:12 (1999), pp. 6682-6688. 